\title{
Metric Regularity of Quasidifferentiable Mappings and Optimality Conditions for Nonsmooth Mathematical Programming Problems
}

\author{
Dolgopolik M.V.*
}

November 19, 2020

\begin{abstract}
This article is devoted to the analysis of necessary and/or sufficient conditions for metric regularity in terms of Demyanov-Rubinov-Polyakova quasidifferentials. We obtain new necessary and sufficient conditions for the local metric regularity of a multifunction in terms of quasidifferentials of the distance function to this multifunction. We also propose a new MFCQ-type constraint qualification for a parametric system of quasidifferentiable equality and inequality constraints and prove that it ensures the metric regularity of a multifunction associated with this system. As an application, we utilize our constraint qualification to strengthen existing optimality conditions for quasidifferentiable programming problems with equality and inequality constraints. We also prove the independence of the optimality conditions of the choice of quasidifferentials and present a simple example in which the optimality conditions in terms of quasidifferentials detect the non-optimality of a given point, while optimality conditions in terms of various subdifferentials fail to disqualify this point as non-optimal.
\end{abstract}

\section{Introduction}

Metric regularity plays a very important role in various parts of optimization theory and numerical analysis, including stability analysis of perturbed optimization problems, subdifferential calculus, analysis of optimality conditions etc. 1, 3, 4, 22, 24, 30, 31 Necessary and/or sufficient conditions for metric regularity are usually expressed in terms of various slopes, subdifferentials and coderivatives 2, 6, 22, 24,30. However, if one studies nonsmooth problems with quasidifferentiable data and wants to utilize quasidifferential calculus [10,11, these conditions for metric regularity become very inconvenient, since one has to compute and use subdifferentials/coderivatives and quasidifferentials simultaneously. In this case it seems more reasonable to apply necessary and/or sufficient

\footnotetext{
*Institute for Problems in Mechanical Engineering, Russian Academy of Sciences, Saint Petersburg, Russia. e-mail: maxim.dolgopolik@gmail.com
} 
conditions for metric regularity in terms of quasidifferentials. Such conditions were studied by Uderzo in $[36$ 38].

One of the main goals of this paper is to improve the main results of $36+38$ and obtain simple conditions for metric regularity in terms of quasidifferentials. With the use of general results on metric regularity 22] we obtain new necessary and sufficient conditions for the metric regularity of multifunctions in terms of quasidifferentials of the distance function to this multifunction (see [15] for some results on the quasidifferentiability of this function). These conditions significantly generalize and improve some results from [36. For example, our conditions, unlike the ones in [36], are invariant under the choice of quasidifferentials. However, both our conditions and the ones in 36 38 have a significant drawback. Namely, one must verify the validity of certain inequalities in a neighbourhood of a given point to apply these conditions. To overcome this issue, we introduce a new MFCQ-type constraint qualification for a parametric system of quasidifferentiable equality and inequality constraint and demonstrate that this constraint qualification guarantees the local metric regularity of a multifunction associated with this system (see 27] for a discussion of constraint qualifications for quasidifferentiable optimization problems with inequality constraints).

As an application, we utilize our constraint qualification to obtain new necessary optimality conditions for quasidifferentiable programming problems with equality and inequality constraints that strengthen existing optimality conditions for these problems in terms of quasidifferentials 33 35. (optimality conditions for such problems involving, e.g. the Demyanov difference of quasidifferentials, can be found in [17). We prove the independence of our optimality conditions of the choice of quasidifferentials (cf. [28, 29]) and present a simple example in which our optimality conditions detect the non-optimality of a given point, while optimality conditions in terms of Clarke, Michel-Penot, Jeyakumar-Luc, Ioffe and Mordukhovich subdifferentials fail to disqualify this point as non-optimal.

The paper is organized as follows. Necessary and sufficient conditions for metric regularity of multifunctions in terms of quasidifferentials are obtained in Section 3. In this section, we also introduce a new MFCQ-type constraint qualification for parametric systems of quasidifferentiable equalities and inequalities and study its connection with metric regularity. This constraint qualification is applied to the derivation of new optimality conditions for quasidifferentiable programming problems in Section 4 Finally, some basic definitions and facts from quasidifferential calculus are collected in Section 2 .

\section{Quasidifferentiable Functions}

From this point onwards let $X$ be a real Banach space. Its topological dual space is denoted by $X^{*}$, whereas the canonical duality pairing between $X$ and $X^{*}$ is denoted by $\langle\cdot, \cdot\rangle$. The zero vector of a vector space $Y$ is denoted by $\mathbb{O}_{Y}$ or simply by $\mathbb{O}$ when the underline space is clear from the context.

Let $U \subset X$ be an open set. Recall that a function $f: U \rightarrow \mathbb{R}$ is called Dini (Hadamard) directionally differentiable at a point $x \in U$, if for any $h \in X$ there 
exists the finite limit

$$
\begin{aligned}
f_{D}^{\prime}(x, h) & =\lim _{\alpha \rightarrow+0} \frac{f(x+\alpha h)-f(x)}{\alpha} \\
\left(f_{H}^{\prime}(x, h)\right. & \left.=\lim _{\left[\alpha, h^{\prime}\right] \rightarrow[+0, h]} \frac{f\left(x+\alpha h^{\prime}\right)-f(x)}{\alpha}\right)
\end{aligned}
$$

(see [18] for a discussion about the limit in the definition of Hadamard directional derivative). Clearly, if $f$ is Hadamard directionally differentiable at $x$, then $f_{H}^{\prime}(x, \cdot)=f_{D}^{\prime}(x, \cdot)$. Therefore, it is natural to refer simply to the directional derivative of $f$ at $x$ and denote it by $f^{\prime}(x, \cdot)$.

Definition 1. A function $f: U \rightarrow \mathbb{R}$ is called Dini (Hadamard) quasidifferentiable at a point $x \in U$ if $f$ is Dini (Hadamard) directionally differentiable at $x$, and its directional derivative can be represented as the difference of two continuous sublinear functions or equivalently if there exists a a pair $\mathscr{D} f(x)=[\underline{\partial} f(x), \bar{\partial} f(x)]$ of convex weak* compact sets $\underline{\partial} f(x), \bar{\partial} f(x) \subset X^{*}$ such that

$$
f^{\prime}(x, h)=\max _{v^{*} \in \underline{\partial} f(x)}\left\langle v^{*}, h\right\rangle+\min _{w^{*} \in \bar{\partial} f(x)}\left\langle w^{*}, h\right\rangle \quad \forall h \in X .
$$

The pair $\mathscr{D} f(x)$ is called a Dini (Hadamard) quasidifferential of $f$ at $x$, while the sets $\underline{\partial} f(x)$ and $\bar{\partial} f(x)$ are called the Dini (Hadamard) subdifferential and superdifferential of $f$ at $x$ respectively.

Remark 1. Following the usual convention we identify $X^{*}$ with $X$ in the case when $X$ is either a finite dimensional or a Hilbert space. Therefore, in particular, if $X=\mathbb{R}^{n}$, then a quasidifferential is a pair of convex compact subsets of $\mathbb{R}^{n}$, while if $X$ is a Hilbert space, then a quasidifferential is a pair of weakly compact convex subsets of $X$.

A calculus of quasidifferentiable functions can be found in [10. Here we only mention that any finite DC (difference-of-convex) function is Hadamard quasidifferentiable. Note also that a quasidifferential of a function $f$ is not unique, since for any quasidifferential $\mathscr{D} f(x)$ of $f$ at $x$ and any weak* compact convex set $C \subset X^{*}$ the pair $[\underline{\partial} f(x)+C, \bar{\partial} f(x)-C]$ is a quasidifferential of $f$ at $x$ as well.

In the general case quasidifferential mapping $\mathscr{D} f(\cdot)$ might not possess any continuity properties; however, for many nonsmooth functions appearing in applications it is outer semicontinuous (o.s.c.). Recall that if a function $f$ is quasidifferentiable in a neighbourhood $U$ of a point $x \in X$, then a quasidifferential mapping $\mathscr{D} f(\cdot)$ defined in this neighbourhood is said to be o.s.c. at $x$, if the corresponding multifunctions $\underline{\partial} f: U \rightarrow X^{*}$ and $\bar{\partial} f: U \rightarrow X^{*}$ are o.s.c. at $x$, i.e. for any open sets $V_{1}, V_{2} \subset X^{*}$ such that $\underline{\partial} f(x) \subset V_{1}$ and $\bar{\partial} f(x) \subset V_{2}$ there exists $\delta>0$ such that $\underline{\partial} f\left(x^{\prime}\right) \subset V_{1}$ and $\bar{\partial} f\left(x^{\prime}\right) \subset V_{2}$ for all $x^{\prime} \in U$ with $\left\|x^{\prime}-x\right\|<\delta$. As was pointed out in [26], a quasidifferential of a continuously codifferentiable function is outer semicontinuous (see [10] for the definition of continuously codifferentiable function). Hence, in particular, the class of functions for which there exists an o.s.c. quasidifferential mapping is closed under all standard algebraic operations, the pointwise maximum and minimum of finite families of functions, and the composition with smooth functions, since the class of continuously codifferentiable functions is closed under all these operations [10,12,14. Furthermore, any DC function has an o.s.c. quasidifferential mapping. Indeed, 
if $f=f_{1}-f_{2}$, where $f_{1}$ and $f_{2}$ are finite closed convex functions, then one can define $\mathscr{D} f(\cdot)=\left[\partial f_{1}(\cdot),-\partial f_{2}(\cdot)\right]$, where $\partial f_{i}(\cdot)$ is the subdifferential of $f_{i}$ in the sense of convex analysis. Note that this quasidifferential is correctly defined and o.s.c. due to the fact that the subdifferential of a finite closed convex function defined on a Banach space is nonempty at every point (see, e.g. 16, Proposition I.5.2. and Corollary I.2.5]) and outer semicontinuous.

Let us also recall a certain extension of the definition of quasidifferentiability to the case of vector-valued functions that was utilized in [19, 37.

Definition 2. Let $Y$ be a real Banach space, and $U \subset X$ be an open set. A function $F: U \rightarrow Y$ is called scalarly quasidifferentible at a point $x \in U$, if $F$ is Dini directionally differentiable at $x$, i.e. for any $h \in X$ there exists the limit

$$
F^{\prime}(x, h)=\lim _{\alpha \rightarrow+0} \frac{1}{\alpha}(F(x+\alpha h)-F(x)),
$$

and for any $y^{*} \in Y^{*}$ the function $\left\langle y^{*}, F^{\prime}(x, \cdot)\right\rangle$ can be represented as the difference of continuous sublinear functions, i.e. there exists a pair of convex weak ${ }^{*}$ compact sets $\underline{\partial} F\left(x ; y^{*}\right), \bar{\partial} F\left(x ; y^{*}\right) \subset X^{*}$ such that

$$
\left\langle y^{*}, F^{\prime}(x, h)\right\rangle=\max _{v^{*} \in \underline{\partial} F\left(x ; y^{*}\right)}\left\langle v^{*}, h\right\rangle+\min _{w^{*} \in \bar{\partial} F\left(x ; y^{*}\right)}\left\langle w^{*}, h\right\rangle \quad \forall v \in X .
$$

For any $y^{*} \in Y^{*}$ the pair $\mathscr{D} F\left(x ; y^{*}\right)=\left[\underline{\partial} F\left(x ; y^{*}\right), \bar{\partial} F\left(x ; y^{*}\right)\right]$ is called a scalar quasidifferential of $F$ at $x$ (corresponding to $y^{*}$ ).

Remark 2. Below, as usual, we use the term "quasidifferential", instead of "Dini quasidifferential". Also, when we say that a function $f$ is quasidifferentiable at a point $x$, we suppose that a quasidifferential of $f$ at $x$ is given. Alternatively, one can define a quasidifferential as an equivalence class and work with these equivalence classes; however, in author's opinion this approach leads to somewhat cumbersome formulations of the main results. That is why we do not adopt it in this article.

\section{Metric Regularity of Quasidifferentiable Map- pings}

In this section we obtain necessary and/or sufficient conditions for the metric regularity of multifunctions in terms of quasidifferentials. We also introduce an MFCQ-type constraint qualifications for parametric systems of quasidifferentiable equalities and inequalities and prove that it ensures the metric regularity of a multifunction associated with this system.

\subsection{General Conditions for Metric Regularity}

Let $(Y, d)$ be a complete metric space, and $F: X \rightrightarrows Y$ be a set-valued mapping with closed values, whose graph is denoted by Graph $F$. For any $y \in Y, r>0$ and any set $C \subset Y$ denote $B(y, r)=\{z \in Y \mid d(y, z) \leq r\}$ and $d(y, C)=$ $\inf _{z \in C} d(y, z)$. As usual, we put $d(y, \emptyset)=+\infty$.

Recall that $F$ is called metrically regular near a point $(\bar{x}, \bar{y}) \in \operatorname{Graph} F$, if there exist $K>0$ and $r>0$ such that

$$
d\left(x, F^{-1}(y)\right) \leq K d(y, F(x)) \quad \forall(x, y) \in B(\bar{x}, r) \times B(\bar{y}, r) .
$$


The greatest lower bound of all $K$ for which the inequality above is satisfied with some $r>0$ is called the norm of metric regularity of $F$ near $(\bar{x}, \bar{y})$. For the general theory of metric regularity see $2,22,24$.

At first, our aim is to obtain sufficient conditions for the metric regularity of the set-valued mapping $F$ in the case when the distance function $x \rightarrow d(y, F(x))$ is quasidifferentiable for any $(x, y)$ in a neighbourhood of $(\bar{x}, \bar{y})$. To this end, for any $y \in Y$ and $x \in X$ denote $\psi_{y}(x)=d(y, F(x))$, and define

$$
\left|\nabla \psi_{y}\right|(x)=\limsup _{u \rightarrow x, \psi_{y}(u) \rightarrow \psi_{y}(x)} \frac{\max \left\{\psi_{y}(x)-\psi_{y}(u), 0\right\}}{\|x-u\|} .
$$

The quantity $\left|\nabla \psi_{y}\right|(x)$ is called the strong slope of $\psi_{y}$ at $x$.

Recall that under some natural assumptions on the functions $\psi_{y}(\cdot)$ the validity of the inequality $\left|\nabla \psi_{y}\right|(x)>K^{-1}$ for any $(x, y) \notin$ Graph $F$ in a neighbourhood of $(\bar{x}, \bar{y})$ is sufficient for the metric regularity of $F$ near $(\bar{x}, \bar{y})$ with the norm of metric regularity no exceeding $K$. In the case when $Y$ is a Banach space, the validity of the inequality $\left|\nabla \psi_{y}\right|(x) \geq t^{-1}$ for any such $(x, y)$ and for all $t>K$ is also necessary for the metric regularity of $F$ near $(\bar{x}, \bar{y})$ with the norm of metric regularity no exceeding $K$ (see, e.g. [22, Theorem $2 \mathrm{~b}]$ ).

In the following theorem we demonstrate how the verification of the inequality $\left|\nabla \psi_{y}\right|(x)>K^{-1}$ (and, thus, the metric regularity of the multifunction $F$ ) can be significantly simplified in the case when the distance functions $\psi_{y}(\cdot)=d(y, F(\cdot))$ are quasidifferentiable.

Theorem 1. Let for any $y \in Y$ the function $\psi_{y}(\cdot)$ be lower semicontinuous (l.s.c.), and let $(\bar{x}, \bar{y}) \in$ Graph $F$ and $K>0$ be given. Suppose that there exists $r>0$ such that for any $(x, y) \in B(\bar{x}, r) \times B(\bar{y}, r)$ with $y \notin F(x)$ the function $\psi_{y}(\cdot)$ is quasidifferentiable at $x$, and there exists $w^{*} \in \bar{\partial} \psi_{y}(x)$ for which

$$
d\left(\mathbb{O}, \underline{\partial} \psi_{y}(x)+w^{*}\right)>\frac{1}{K} .
$$

Then for any $(x, y) \in B(\bar{x}, r) \times B(\bar{y}, r)$ such that $K d(y, F(x))<r-d(x, \bar{x})$ one has $d\left(x, F^{-1}(y)\right) \leq K d(y, F(x))$, which, in particular, implies that the set-valued mapping $F$ is metrically regular near $(\bar{x}, \bar{y})$ with the norm of metric regularity not exceeding $K$.

Moreover, suppose that $Y$ is a Banach space, $X$ is finite dimensional, and for any $y \in Y$ the functions $\psi_{y}(\cdot)$ are Hadamard quasidifferentiable on $B(\bar{x}, r)$ with some $r>0$. Then for the metric regularity of $F$ near $(\bar{x}, \bar{y})$ with the norm of metric regularity not exceeding $K$ it is necessary and sufficient that for any $t>$ $K$ there exists a neighbourhood $U$ of $(\bar{x}, \bar{y})$ such that for any $(x, y) \in U \backslash \operatorname{Graph} F$ there exists $w^{*} \in \bar{\partial} \psi_{y}(x)$ for which $d\left(\mathbb{O}, \underline{\partial} \psi_{y}(x)+w^{*}\right) \geq t^{-1}$.

Proof. Let us show that under the assumptions of the theorem one has

$$
\left|\nabla \psi_{y}\right|(x)>K^{-1} \quad \forall(x, y) \in(B(\bar{x}, r) \times B(\bar{y}, r)) \backslash \operatorname{Graph} F .
$$

Then applying 22 , Theorem $2 \mathrm{~b}]$ one obtains the desired result.

Indeed, fix $(x, y) \in B(\bar{x}, r) \times B(\bar{y}, r)$ with $y \notin F(x)$. From (4) it follows that for some $\varepsilon>0$ the convex compact subsets $B\left(\mathbb{O}, K^{-1}+\varepsilon\right)$ and $\underline{\partial} \psi_{y}(x)+w^{*}$ 
of the space $X^{*}$ endowed with the weak* topology are disjoint. Applying the separation theorem one obtains that there exists $h \in X$ with $\|h\|=1$ such that

$$
\left\langle v^{*}, h\right\rangle \leq\left\langle x^{*}, h\right\rangle \quad \forall v^{*} \in \underline{\partial} \psi_{y}(x)+w^{*} \quad \forall x^{*} \in B\left(\mathbb{O}, K^{-1}+\varepsilon\right)
$$

or equivalently $\left\langle v^{*}, h\right\rangle \leq-K^{-1}-\varepsilon<-K^{-1}$ for any $v^{*} \in \underline{\partial} \psi_{y}(x)+\left\{w^{*}\right\}$. Hence

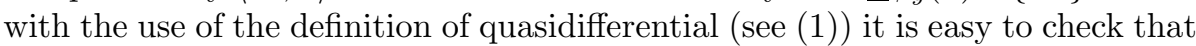
$\psi_{y}^{\prime}(x, h)<K^{-1}$. Therefore there exists a sequence $\left\{\alpha_{n}\right\} \subset(0,+\infty)$ such that $\alpha_{n} \rightarrow 0$ as $n \rightarrow \infty$ and

$$
\lim _{n \rightarrow \infty} \frac{\psi_{y}\left(x+\alpha_{n} h\right)-\psi_{y}(x)}{\alpha_{n}}<-\frac{1}{K} .
$$

Consequently, $\psi_{y}(x)-\psi_{y}\left(x+\alpha_{n} h\right)>0$ for any sufficiently large $n \in \mathbb{N}$, and

$$
\limsup _{n \rightarrow \infty} \frac{\max \left\{\psi_{y}(x)-\psi_{y}\left(x+\alpha_{n} h\right), 0\right\}}{\alpha_{n}}>\frac{1}{K},
$$

which due to (3) yields $\left|\nabla \psi_{y}\right|(x)>K^{-1}$, since $\|h\|=1$.

Let us now prove the second part of the theorem. Indeed, by [22, Theorem $2 \mathrm{~b}]$ the multifunction $F$ is metrically regular near $(\bar{x}, \bar{y})$ with the norm of metric regularity not exceeding $K$ iff for any $t>K$ there exists a neighbourhood $U$ of $(\bar{x}, \bar{y})$ such that $\left|\nabla \psi_{y}\right|(x) \geq t^{-1}$ for any $(x, y) \in U \backslash$ Graph $F$.

Taking into account the facts that $X$ is finite dimensional and the functions $x \rightarrow \psi_{y}(x)$ are Hadamard quasidifferentiable, and applying [2, Proposition 2.8] one obtains that $\left|\nabla \psi_{y}\right|(x)=-\min _{\|h\|=1} \psi_{y}^{\prime}(x, h)$. Hence with the use of the explicit expression for the rate of steepest descent of a quasidifferentiable function (see [10, Section V.3.1]) one gets

$$
\left|\nabla \psi_{y}\right|(x)=\max _{w^{*} \in \bar{\partial} \psi_{y}(x)} \min _{v^{*} \in \underline{\partial} \psi_{y}(x)+\left\{w^{*}\right\}}\left\|v^{*}\right\|, \quad \text { if }\left|\nabla \psi_{y}\right|(x)>0 .
$$

Consequently, $\left|\nabla \psi_{y}\right|(x) \geq t^{-1}$ iff $d\left(\mathbb{O}, \underline{\partial} \psi_{y}(x)+w^{*}\right) \geq t^{-1}$ for some $w^{*} \in \bar{\partial} \psi_{y}(x)$, which implies the required result.

Remark 3. Taking into account the definition of quasidifferential (11) it is easy to check that condition (4) is satisfied for some $w^{*} \in \bar{\partial} \psi_{y}(x)$ iff there exists $h \in X$ with $\|h\|=1$ such that $\psi_{y}^{\prime}(x, h)<-K^{-1}$. Therefore, condition (4) is invariant with respect to the choice of quasidifferentials of the functions $\psi_{y}$, since the directional derivative $\psi_{y}^{\prime}(x, \cdot)$ obviously does not depend on the choice of quasidifferential.

Remark 4. Sufficient conditions for the metric regularity of a continuous singlevalued mapping $F$ between Banach spaces in terms of quasidifferentials of the functions $\psi_{y}(x)=\|y-F(x)\|$ were first obtained by Uderzo [36] (see also [37]). However, the conditions in [36] are more restrictive then the ones stated in the theorem above. Indeed, by [36, Theorem 4.3] for the metric regularity of $F$ near a point $(\bar{x}, F(\bar{x}))$ it is sufficient that there exist $m>0$ and $r>0$ such that for any $x \in B(\bar{x}, r)$ and $y \in B(F(\bar{x}), r)$ with $y \neq F(x)$ one has

$$
d\left(\mathbb{O}, \underline{\partial} \psi_{y}(x)+w^{*}\right)>m \quad \forall w^{*} \in \bar{\partial} \psi_{y}(x) .
$$

It is easy to see that this condition fails to hold true even for the very simple function $F\left(x_{1}, x_{2}\right)=\left|x_{1}\right|-\left|x_{2}\right|$, when $\bar{x}=\mathbf{0}_{2}$ and $\bar{y}=0$ (here $X=\mathbb{R}^{2}, Y=\mathbb{R}$, 
and $\mathbf{0}_{n}$ is the zero vector from $\left.\mathbb{R}^{n}\right)$. Indeed, for $x=\mathbf{0}_{2}$ and any $y>0$ a quasidifferential of the function $\psi_{y}(x)=|y-F(x)|$ has the form

$$
\underline{\partial} \psi_{y}\left(\mathbf{0}_{2}\right)=\operatorname{co}\left\{\left(\begin{array}{l}
0 \\
1
\end{array}\right),\left(\begin{array}{c}
0 \\
-1
\end{array}\right)\right\}, \quad \bar{\partial} \psi_{y}\left(\mathbf{0}_{2}\right)=\operatorname{co}\left\{\left(\begin{array}{l}
1 \\
0
\end{array}\right),\left(\begin{array}{c}
-1 \\
0
\end{array}\right)\right\},
$$

and for $w^{*}=\mathbf{0}_{2} \in \bar{\partial} \psi_{y}\left(\mathbf{0}_{2}\right)$ one has $\mathbf{0}_{2} \in \underline{\partial} \psi_{y}\left(\mathbf{0}_{2}\right)+w^{*}$. Thus, condition (5) is not satisfied. On the other hand, one can check that sufficient conditions from Theorem 1 are satisfied. Indeed, fix any $x \in \mathbb{R}^{2}$ and $y \in \mathbb{R}$ such that $y \neq F(x)$. Applying standard rules of quasidifferential calculus [10, Section III.2] to the function $\psi_{y}(x)=|y-| x_{1}|+| x_{2}||$ one gets that

$$
\underline{\partial} \psi_{y}(x)=\left\{\left(\begin{array}{c}
0 \\
\operatorname{Sign}\left(x_{2}\right)
\end{array}\right)\right\}, \quad \bar{\partial} \psi_{y}(x)=\left\{\left(\begin{array}{c}
-\operatorname{Sign}\left(x_{1}\right) \\
0
\end{array}\right)\right\}
$$

in the case $y>\left|x_{1}\right|-\left|x_{2}\right|$, and

$$
\underline{\partial} \psi_{y}(x)=\left\{\left(\begin{array}{c}
\operatorname{Sign}\left(x_{1}\right) \\
0
\end{array}\right)\right\}, \quad \bar{\partial} \psi_{y}(x)=\left\{\left(\begin{array}{c}
0 \\
-\operatorname{Sign}\left(x_{2}\right)
\end{array}\right)\right\}
$$

in the case $y<\left|x_{1}\right|-\left|x_{2}\right|$. Here $\operatorname{Sign}(t)=\operatorname{sign}(t)$, if $t \neq 0$, and $\operatorname{Sign}(0)=[-1,1]$. Let the space $X=\mathbb{R}^{2}$ be equipped with the Euclidean norm. Then we have the following two cases:

1. if $y>F(x)$, then setting $w^{*}=\left(-\operatorname{sign}\left(x_{1}\right), 0\right)^{T} \in \bar{\partial} \psi_{y}(x)$ in the case $x_{1} \neq 0$ and $w^{*}=(1,0)^{T} \in \bar{\partial} \psi_{y}(x)$ in the case $x_{1}=0$ one gets that $d\left(\mathbf{0}_{2}, \underline{\partial} \psi_{y}(x)+w^{*}\right)=\sqrt{2}$, provided $x_{2} \neq 0$, and $d\left(\mathbf{0}_{2}, \underline{\partial} \psi_{y}(x)+w^{*}\right)=1$, if $x_{2}=0$

2. if $y<F(x)$, then setting $w^{*}=\left(0,-\operatorname{sign}\left(x_{2}\right)\right)^{T} \in \bar{\partial} \psi_{y}(x)$ in the case $x_{2} \neq 0$ and $w^{*}=(0,1) \in \bar{\partial} \psi_{y}(x)$ in the case $x_{2}=0$ one obtains that $d\left(\mathbf{0}_{2}, \underline{\partial} \psi_{y}(x)+w^{*}\right)=\sqrt{2}$, if $x_{1} \neq 0$, and $d\left(\mathbf{0}_{2}, \underline{\partial} \psi_{y}(x)+w^{*}\right)=1$, if $x_{1}=0$.

Thus, condition (4) is satisfied with any $K>1$, and the function $F(x)=$ $\left|x_{1}\right|-\left|x_{2}\right|$ is metrically regular near the point $\left(\mathbf{0}_{2}, 0\right)$ by Theorem 1.

Note also that condition (5), unlike (4), depends on the choice of quasidifferential. For instance, it is not valid for the identity function $F(x)=x$, which is metrically regular near any point (here $X=Y=\mathbb{R}$ ), if one chooses the pair $\underline{\partial} \psi_{y}(x)=-\operatorname{sign}(y-x)+[-1,1]$ and $\bar{\partial} \psi_{y}(x)=[-1,1]$, as a quasidifferential of the function $\psi_{y}(x)=|y-F(x)|=|y-x|$ at every point $x$ such that $y \neq x$.

Let us give another simple example illustrating Theorem 1

Example 1. Let $X=Y=\mathbb{R}$ and $F(x)=\min \left\{x, \max \left\{x^{3}, 0\right\}\right\}$, i.e. $F$ is single-valued. Let us check whether this function is metrically regular near the point $(0,0)$ with the use of Theorem 1. By definition one has $\psi_{y}(x)=$ $\left|y-\min \left\{x, \max \left\{x^{3}, 0\right\}\right\}\right|$. The function $\psi_{y}(\cdot)$ is quasidifferentiable and locally Lipschitz continuous, which implies that it is Hadamard quasidifferentiable. Applying standard rules of quasidifferential calculus [10, Section III.2] one gets 
that

$$
\begin{aligned}
& \underline{\partial} \psi_{y}(x)= \begin{cases}\{-1\}, & \text { if } x \notin[0,1], \\
\left\{-3 x^{2}\right\}, & \text { if } x \in(0,1), \\
{[-3,-1],} & \text { if } x=1, \\
{[-1,0],} & \text { if } x=0\end{cases} \\
& \underline{\partial} \psi_{y}(x)=\{0\}, \quad \bar{\partial} \psi_{y}(x)=\left\{\begin{array}{ll}
\{1\}, & \text { if } x \notin[0,1], \\
\left\{3 x^{2}\right\}, & \text { if } x \in(0,1), \\
{[1,3],} & \text { if } x=1, \\
{[0,1],} & \text { if } x=0
\end{array} \text { in the case } y<F(x) .\right.
\end{aligned}
$$

Therefore for $y=0$ and any $x \in(0,1)$ (note that in this case $y \neq F(x)$ ) one has $d\left(0, \underline{\partial} \psi_{y}(x)+w^{*}\right)=3 x^{2}$ for any $w^{*} \in \bar{\partial} \psi_{y}(x)$. Choosing sufficiently small $x>0$ one obtains that $d\left(0, \underline{\partial} \psi_{0}(x)+w^{*}\right)<t^{-1}$ for any prespecified $t>0$ and for all $w^{*} \in \bar{\partial} \psi_{0}(x)$. Thus, by the second part of Theorem 1 one can conclude that $F$ is not metrically regular near $(0,0)$. Let us also verify this directly. Indeed, it is easily seen that $F^{-1}(y)=y$, if $y \notin[0,1]$, and $F^{-1}(y)=y^{1 / 3}$, if $y \in[0,1]$. Applying the definition of metric regularity (2) with $x=0$ one gets that for the function $F$ to be metrically regular near $(0,0)$ it is necessary that there exists $K>0$ such that

$$
d\left(0, F^{-1}(y)\right)=y^{1 / 3} \leq K y=d(y, F(0))
$$

for any sufficiently small $y>0$, which is obviously impossible.

\subsection{Parametric Systems of Equalities and Inequalities}

In order to verify the metric regularity of a multifunction with the use of Theorem 1, one must check that condition (4) holds true at every point in a neighbourhood of a given point $(\bar{x}, \bar{y})$, which is a common drawback of general results on metric regularity (cf. [2, 22]). However, as in the case of sufficient conditions in terms of various subdifferentials and coderivatives, in some particular cases one can obtain sufficient conditions for the metric regularity that involve only quasidifferentials of certain functions at the point $(\bar{x}, \bar{y})$ itself. Our next goal is to obtain such conditions for a set-valued mapping associated with a parametric system of nonlinear equality and inequality constraints.

Let $Y$ be a real Banach space, $P$ be a metric space of parameters, and let also $F: X \times P \rightarrow Y$ and $g_{i}: X \times P \rightarrow \mathbb{R}, i \in I=\{1, \ldots, m\}$, be given functions. For any $y \in Y$ and $z_{i} \in \mathbb{R}, i \in I$, consider the following parametric system

$$
F(x, p)=y, \quad g_{i}(x, p) \leq z_{i} \quad i \in I
$$

Denote by $\mathcal{S}(p, y, z)=\left\{x \in X \mid F(x, p)=y, g_{i}(x, p) \leq z_{i}, i \in I\right\}$ the solution set of this system, where $z=\left(z_{1}, \ldots, z_{m}\right)^{T} \in \mathbb{R}^{m}$. We also denote $\mathcal{S}(p)=$ $\mathcal{S}\left(p, \mathbb{O}_{Y}, \mathbf{0}_{m}\right)$, and sometimes use the notation $F_{p}(x)=F(x, p)$.

In the case when the functions $F(\cdot, p)$ and $g_{i}(\cdot, p)$ are continuously Fréchet differentiable, the multifunction $\Phi_{p}(x)=\{F(x, p)\} \times \prod_{i=1}^{m}\left[g_{i}(x, p),+\infty\right)$ associated with system (6) is metrically regular near a given point if and only if the Mangasarian-Fromovitz constrain qualification holds at this point, i.e. the Fréchet derivative $D_{x} F(x, p)$ is a surjective mapping, and there exists $h \in X$ 
such that $D_{x} F(x, p)[h]=\mathbb{O}$, while $D_{x} g_{i}(x, p)[h]<0$ for any $i \in I$ such that $g_{i}(x, p)=z_{i}$ (see, e.g. [6. Corollary 2.1]). Our aim is to extend this results to the case when the functions $F(\cdot, p)$ and $g_{i}(\cdot, p)$ are only quasidifferentiable.

Being inspired by the results of [37, let us introduce a constraint qualification in terms of quasidifferentials that ensures the metric regularity of the multifunction associated with system (6). For the sake of shortness we consider the case $y=\mathbb{O}$ and $z=\mathbf{0}_{m}$ only, since the general case can be easily reduced to this one by replacing $F(x, p)$ with $F(x, p)-y$, and $g_{i}(x, p)$ with $g_{i}(x, p)-z_{i}$. Suppose that the functions $g_{i}(\cdot, \bar{p}), i \in I$, are quasidifferentiable at a point $\bar{x}$ such that $\bar{x} \in \mathcal{S}(\bar{p})$, and the mapping $F(\cdot, \bar{p})$ is scalarly quasidifferentiable at this point, and denote their quasidifferentials at this point by $\mathscr{D}_{x} g_{i}(\bar{x}, \bar{p})$ and $\mathscr{D}_{x} F\left(\bar{x}, \bar{p} ; y^{*}\right), y^{*} \in Y^{*}$, respectively. Introduce the sets

$$
\begin{aligned}
{\left[\mathscr{D}_{x} g_{i}(\bar{x}, \bar{p})\right]^{+} } & =\underline{\partial}_{x} g_{i}(\bar{x}, \bar{p})+\bar{\partial}_{x} g_{i}(\bar{x}, \bar{p}), \\
{\left[\mathscr{D}_{x} F\left(\bar{x}, \bar{p} ; y^{*}\right)\right]^{+} } & =\underline{\partial}_{x} F\left(\bar{x}, \bar{p} ; y^{*}\right)+\bar{\partial}_{x} F\left(\bar{x}, \bar{p} ; y^{*}\right) .
\end{aligned}
$$

These sets are sometimes called quasidifferential sums, and they were considered e.g. in 37. Note that quasidifferential sums are not invariant with respect to the choice of the corresponding quasidifferentials. For example, for the function $f(x)=|x|$ both $\mathscr{D}_{1} f(0)=[[-1,1],\{0\}]$ and $\mathscr{D}_{2} f(0)=[[-2,2],[-1,1]]$ are quasidifferentials of $f$ at $x$, and $\left[\mathscr{D}_{1} f(0)\right]^{+}=[-1,1] \neq[-3,3]=\left[\mathscr{D}_{2} f(0)\right]^{+}$. Thus, all conditions below are not invariant with respect to the choise of quasidifferentials.

For any $x \in X$ and $p \in P$ define $I(x, p)=\left\{i \in I \mid g_{i}(x, p)=0\right\}$, and denote $S_{X}=\{x \in X \mid\|x\|=1\}$.

Definition 3. One says that the Mangasarian-Fromovitz constraint qualification in terms of quasidifferentials (q.d.-MFCQ) holds at $(\bar{x}, \bar{p})$, if

$$
\inf _{y^{*} \in S_{Y^{*}}} \inf \left\{\left\|v^{*}\right\|: v^{*} \in\left[\mathscr{D}_{x} F\left(\bar{x}, \bar{p} ; y^{*}\right)\right]^{+}\right\}>0,
$$

and there exists $\bar{h} \in X$ such that $\left\langle v^{*}, \bar{h}\right\rangle=0$ for all $v^{*} \in\left[\mathscr{D}_{x} F\left(\bar{x}, \bar{p} ; y^{*}\right)\right]^{+}$and $y^{*} \in Y^{*}$, while $\left\langle v^{*}, \bar{h}\right\rangle<0$ for all $v^{*} \in\left[\mathscr{D}_{x} g_{i}(\bar{x}, \bar{p})\right]^{+}$and $i \in I(\bar{x}, \bar{p})$.

Let us point out how q.d.-MFCQ is connected with the standard MFCQ. To this end, recall that nonempty subsets $A_{1}, \ldots, A_{s}$ of a linear space $E$ are said to be linearly independent (or to have full rank), if the inclusion $\mathbb{O} \in$ $\lambda_{1} A_{1}+\ldots+\lambda_{n} A_{n}$ with $\lambda_{i} \in \mathbb{R}$ is valid only for $\lambda_{i}=0, i \in\{1, \ldots, s\}$. Clearly, the sets $A_{i}, i \in\{1, \ldots, s\}$ are linearly independent iff for any $x_{i} \in A_{i}, i \in\{1, \ldots, s\}$, the vectors $x_{1}, \ldots, x_{s}$ are linearly independent.

Proposition 1. Let $Y$ be the space $\mathbb{R}^{l}$ equipped with the Euclidean norm $|\cdot|$, and $F(\cdot)=\left(f_{1}(\cdot), \ldots, f_{l}(\cdot)\right)^{T}$, where the functions $f_{j}: X \times P \rightarrow \mathbb{R}$ are quasidifferentiable in $x$ at $(\bar{x}, \bar{p})$. Then the mapping $F(\cdot, \bar{p})$ is scalarly quasidifferentiable at $\bar{x}$. Moreover, q.d.-MFCQ holds at $(\bar{x}, \bar{p})$ iff the sets $\left[\mathscr{D}_{x} f_{j}(\bar{x}, \bar{p})\right]^{+}, 1 \leq j \leq l$, are linearly independent, and there exists $\bar{h} \in X$ such that $\left\langle v^{*}, \bar{h}\right\rangle=0$ for all $v^{*} \in\left[\mathscr{D}_{x} f_{j}(\bar{x}, \bar{p})\right]^{+}$and $1 \leq j \leq l$, while $\left\langle v^{*}, \bar{h}\right\rangle<0$ for all $v^{*} \in\left[\mathscr{D}_{x} g_{i}(\bar{x}, \bar{p})\right]^{+}$ and $i \in I(\bar{x}, \bar{p})$.

Proof. From the fact that the functions $f_{j}(\cdot, \bar{p})$ are quasidifferentiable at $\bar{x}$ it follows that the mapping $F(\cdot, \bar{p})$ is directionally differentiable at this point, and

$$
[F(\cdot, \bar{p})]^{\prime}(\bar{x}, h)=\left(\left[f_{1}(\cdot, \bar{p})\right]^{\prime}(\bar{x}, h), \ldots,\left[f_{l}(\cdot, \bar{p})\right]^{\prime}(\bar{x}, h)\right)^{T}
$$


for any $h \in X$. Therefore, for any $y^{*}=\left(y_{1}, \ldots, y_{l}\right)^{T} \in \mathbb{R}^{l}$ one has

$$
\left\langle y^{*},[F(\cdot, \bar{p})]^{\prime}(\bar{x}, h)\right\rangle=\sum_{j=1}^{l} y_{j}\left(\max _{v^{*} \in \underline{\underline{\partial}}_{x} f_{j}(\bar{x}, \bar{p})}\left\langle v^{*}, h\right\rangle+\min _{w^{*} \in \overline{\bar{\partial}}_{x} f_{j}(\bar{x}, \bar{p})}\left\langle w^{*}, h\right\rangle\right),
$$

which implies that $F(\cdot, \bar{p})$ is scalarly quasidifferentiable at $\bar{x}$, and for any $y^{*}$ one can define

$$
\begin{aligned}
& \underline{\partial}_{x} F\left(\bar{x}, \bar{p} ; y^{*}\right)=\sum_{j=1}^{l}\left(\left[y_{j}\right]_{+} \underline{\partial}_{x} f_{j}(\bar{x}, \bar{p})-\left[-y_{j}\right]_{+} \bar{\partial}_{x} f_{j}(\bar{x}, \bar{p})\right), \\
& \bar{\partial}_{x} F\left(\bar{x}, \bar{p} ; y^{*}\right)=\sum_{j=1}^{l}\left(\left[y_{j}\right]_{+} \bar{\partial}_{x} f_{j}(\bar{x}, \bar{p})-\left[-y_{j}\right]_{+} \underline{\partial}_{x} f_{j}(\bar{x}, \bar{p})\right),
\end{aligned}
$$

where $[t]_{+}=\max \{t, 0\}$ for any $t \in \mathbb{R}$. Hence for any $y^{*}$ one has

$$
\left[\mathscr{D}_{x} F\left(\bar{x}, \bar{p} ; y^{*}\right)\right]^{+}=\sum_{j=1}^{l} y_{j}\left[\mathscr{D}_{x} f_{j}(\bar{x}, \bar{p})\right]^{+} .
$$

Consequently, if (7) holds true, then the sets $\left[\mathscr{D}_{x} f_{j}(\bar{x}, \bar{p})\right]^{+}, 1 \leq j \leq l$, are linearly independent, since otherwise $0 \in\left[\mathscr{D}_{x} F\left(\bar{x}, \bar{p} ; y^{*}\right)\right]$ for $y^{*}=\lambda /|\lambda|$, where $\lambda \in \mathbb{R}^{l}$, $\lambda \neq \mathbf{0}_{l}$ is such that $\mathbb{O} \in \lambda_{1}\left[\mathscr{D}_{x} f_{1}(\bar{x}, \bar{p})\right]^{+}+\ldots+\lambda_{l}\left[\mathscr{D} f_{l}(\bar{x}, \bar{p})\right]^{+}$, which is impossible. Conversely, if the sets $\left[\mathscr{D}_{x} f_{j}(\bar{x}, \bar{p})\right]^{+}, 1 \leq j \leq l$, are linearly independent, then $\mathbb{O} \notin\left[\mathscr{D}_{x} F\left(\bar{x}, \bar{p} ; y^{*}\right)\right]^{+}$for any $y^{*} \neq \mathbf{0}_{l}$. Applying the separation theorem and the fact that the set $\left[\mathscr{D}_{x} F\left(\bar{x}, \bar{p} ; y^{*}\right)\right]^{+}$is weak* compact one obtains that there exist $h \in X$ and $\delta>0$ such that $\left\langle v^{*}, h\right\rangle \geq \delta$ for all $v^{*} \in\left[\mathscr{D}_{x} F\left(\bar{x}, \bar{p} ; y^{*}\right)\right]^{+}$. Therefore $\inf \left\{\left\|v^{*}\right\| \mid v^{*} \in\left[\mathscr{D}_{x} F\left(\bar{x}, \bar{p} ; y^{*}\right)\right]^{+}\right\}>0$ for any $y^{*} \neq \mathbf{0}_{l}$. Hence taking into account the facts that this infimum is obviously continuous with respect to $y^{*}$ (see (8)), and the unit sphere in $\mathbb{R}^{l}$ is compact one gets that (7) holds true. It remains to note that the equivalence between the second conditions from q.d.-MFCQ and the proposition (the existence of $\bar{h}$ ) follows directly from (8).

Remark 5. With the use of the separation theorem one can easily check that under the assumptions of the proposition above the vector $\bar{h}$ from q.d.-MFCQ exists iff

$$
\operatorname{co}\left\{\left[\mathscr{D}_{x} g_{i}(\bar{x}, \bar{p})\right]^{+} \mid i \in I(\bar{x}, \bar{p})\right\} \cap \operatorname{cl} \operatorname{span}\left\{\left[\mathscr{D}_{x} f_{j}(\bar{x}, \bar{p})\right]^{+} \mid 1 \leq j \leq l\right\}=\emptyset,
$$

where the closure is taken in the weak* topology. Furthermore, if $X$ is finite dimensional, then this span is weak ${ }^{*}$ closed, and (9) is equivalent to the following condition: for any $v_{i}^{*} \in\left[\mathscr{D}_{x} g_{i}(\bar{x}, \bar{p})\right]^{+}, i \in I(\bar{x}, \bar{p})$, and $w_{k}^{*} \in \bigcup_{1 \leq j \leq l}\left[\mathscr{D}_{x} f_{j}(\bar{x}, \bar{p})\right]^{+}$, $1 \leq k \leq n$, where $n$ is the dimension of $X$, there exists $\bar{h} \in X$ such that

$$
\left\langle v_{i}^{*}, \bar{h}\right\rangle<0 \quad \forall i \in I(\bar{x}, \bar{p}), \quad\left\langle w_{k}^{*}, \bar{h}\right\rangle=0 \quad \forall k \in\{1, \ldots, n\} .
$$

The implication (9) $\Longrightarrow$ (10) follows from the separation theorem, while the opposite implication follows from the fact that if the intersection in (9) is not empty, then it is impossible to find $\bar{h}$ satisfying (10) for those $v_{i}^{*}$ and $w_{k}^{*}$ that correspond to a vector from the intersection. Note that condition (10) is, in a sense, a "pointwise" version of the second condition from q.d.-MFCQ. Let us finally point out that in the case when $l=1$ the "linear independence condition" from q.d.-MFCQ is reduced to $\mathbb{O} \notin\left[\mathscr{D}_{x} f_{1}(\bar{x}, \bar{p})\right]^{+}$. 
Likewise the standard Mangasarian-Fromowitz constraint qualification, q.d.MFCQ can be used to obtain sufficient conditions for metric regularity. For the sake of simplicity we consider only the case when the functions $F$ and $g_{i}$ are continuous on $X \times P$, although the theorem below holds true under weaker assumptions. Note also that in the theorem below, unlike in the main results of [37, we do not assume that the Banach space $Y$ admits a Fréchet smooth renorming.

Theorem 2. Suppose that the functions $F$ and $g_{i}, i \in I$, are continuous. Let also a point $(\bar{x}, \bar{p}) \in X \times P$ be such that $\bar{x} \in \mathcal{S}(\bar{p})$, and there exist a neighbourhood $U$ of $(\bar{x}, \bar{p})$ such that

1. for any $(x, p) \in U$ the mapping $F(\cdot, p)$ is scalarly quasidifferentiable at $x$, and the functions $g_{i}(\cdot, p), i \in I(\bar{x}, \bar{p})$, are quasidifferentiable at $x$;

2. the multifunctions $\mathscr{D}_{x} g_{i}(\cdot), i \in I(\bar{x}, \bar{p})$, are o.s.c. at $(\bar{x}, \bar{p})$, while the multifunction $(x, p) \mapsto\left[\mathscr{D}_{x} F\left(x, p ; y^{*}\right)\right]^{+}$is o.s.c. at $(\bar{x}, \bar{p})$ uniformly with respect to $y^{*} \in S_{Y^{*}}$, i.e. for any $\varepsilon>0$ there exists $\delta>0$ such that $\left[\mathscr{D}_{x} F\left(x, p ; y^{*}\right)\right]^{+} \subseteq\left[\mathscr{D}_{x} F\left(\bar{x}, \bar{p} ; y^{*}\right)\right]^{+}+B(\mathbb{O}, \varepsilon)$ for all $y^{*} \in S_{Y^{*}}$ and $(x, p) \in B(\bar{x}, \delta) \times B(\bar{p}, \delta)$;

3. the set $D(y)=\left\{\left[\mathscr{D}_{x} F\left(\bar{x}, \bar{p} ; y^{*}\right)\right]^{+} \mid y^{*} \in S_{Y^{*}}:\left\langle y^{*}, y\right\rangle=\|y\|\right\}$ is weak closed and convex for any $y \in S_{Y}$.

Suppose, finally, that q.d.-MFCQ holds at $(\bar{x}, \bar{p})$. Then there exist $K>0$, a neighbourhood $V$ of $(\bar{x}, \bar{p})$, and a neighbourhood $W$ of zero in $Y \times \mathbb{R}^{m}$ such that

$$
d(x, \mathcal{S}(p, y, z)) \leq K\left(\|F(x, p)-y\|+\sum_{i=1}^{m} \max \left\{g_{i}(x, p)-z_{i}, 0\right\}\right)
$$

for all $(x, p) \in V$ and $(y, z) \in W$. Therefore, in particular, the set-valued mapping $\Phi_{p}: X \rightrightarrows Y \times \mathbb{R}^{m}, \Phi_{p}(x)=\{F(x, p)\} \times \prod_{i=1}^{m}\left[g_{i}(x, p),+\infty\right)$ is metrically regular near the point $\left(\bar{x},\left(\mathbb{O}_{Y}, \mathbf{0}_{m}\right)\right)$ with the norm of metric regularity not exceeding $K$ for all $p$ in a neighbourhood of $\bar{p}$.

Proof. Let $\bar{r}>0$ be such that $B(\bar{x}, \bar{r}) \times B(\bar{p}, \bar{r}) \subset U$. Our aim is to prove that there exist $r \in(0, \bar{r})$ and $K>0$ such that for any $p \in B(\bar{p}, r)$ one has $\left|\nabla \psi_{(y, z, p)}\right|(x)>K^{-1}$ for all $(y, z) \in B\left(\left(\mathbb{O}_{Y}, \mathbf{0}_{m}\right), r\right)$ and $x \in B(\bar{x}, r)$ such that $(y, z) \notin \Phi_{p}(x)$, where $\psi_{(y, z, p)}(x)=d\left((y, z), \Phi_{p}(x)\right)$, and the space $Y \times \mathbb{R}^{m}$ is equipped with the norm $\|(y, z)\|=\|y\|+\sum_{i=1}^{m}\left|z_{i}\right|$. Then applying [22, Theorem 2b] one obtains that $d\left(x, \Phi_{p}^{-1}(y, z)\right) \leq K d\left((y, z), \Phi_{p}(x)\right)$ for all $x \in B(\bar{x}, r)$, $p \in B(\bar{p}, r)$, and $(y, z) \in B\left(\left(\mathbb{O}_{Y}, \mathbf{0}_{m}\right), r\right)$ such that $K d\left((y, z), \Phi_{p}(x)\right)<r-\| x-$ $\bar{x} \|$, i.e. (11) holds true for all such $x, p, y$, and $z$. With the use of the continuity of the functions $F$ and $g_{i}$ and the fact that $\bar{x} \in \mathcal{S}(\bar{p})$, i.e. $\left(\mathbb{O}_{Y}, \mathbf{0}_{m}\right) \in \Phi_{\bar{p}}(\bar{x})$, one can find $\delta<r$ such that $K d\left((y, z), \Phi_{p}(x)\right)<r-\|x-\bar{x}\|$ for all $x \in B(\bar{x}, \delta)$, $p \in B(\bar{p}, \delta)$ and $(y, z) \in B\left(\left(\mathbb{O}_{Y}, \mathbf{0}_{m}\right), \delta\right)$, which implies that (11) holds true for all such $x, p, y$, and $z$, and the proof is complete.

Before we proceed to the proof of the inequality $\left|\nabla \psi_{(y, z, p)}\right|(x)>K^{-1}$, let us first compute the directional derivative of the mapping $\|F(\cdot, p)-y\|$. Denote $\omega(y)=\|y\|$. Recall that $\partial \omega(y)=\left\{y^{*} \in S_{Y^{*}} \mid\|y\|=\left\langle y^{*}, y\right\rangle\right\}$ for any $y \neq \mathbb{O}$, where $\partial \omega(y)$ is the subdifferential of $\omega$ at $y$ in the sense of convex analysis. 
Fix $(x, p) \in U$ and $y \in Y$. From the definition of scalar quasidifferentiability it follows that for any $h \in X$ one has

$$
F_{p}(x+\alpha h)-F_{p}(x)=\alpha F_{p}^{\prime}(x, h)+o(\alpha) \quad \forall \alpha \geq 0,
$$

where $\|o(\alpha)\| / \alpha \rightarrow 0$ as $\alpha \rightarrow+0$ (recall that $F_{p}(x)=F(x, p)$ ). Hence

$$
\begin{aligned}
& \left|\left\|F_{p}(x+\alpha h)-y\right\|-\left\|F_{p}(x)-y\right\|-\alpha \omega^{\prime}\left(F_{p}(x)-y, F_{p}^{\prime}(x, h)\right)\right| \\
= & \left|\left\|F_{p}(x)-y+\alpha F_{p}^{\prime}(x, h)+o(\alpha)\right\|-\left\|F_{p}(x)-y\right\|-\alpha \omega^{\prime}\left(F_{p}(x)-y, F_{p}^{\prime}(x, h)\right)\right| \\
\leq & \left|\left\|F_{p}(x)-y+\alpha F_{p}^{\prime}(x, h)\right\|-\left\|F_{p}(x)-y\right\|-\alpha \omega^{\prime}\left(F_{p}(x)-y, F_{p}^{\prime}(x, h)\right)\right|+\|o(\alpha)\| .
\end{aligned}
$$

Dividing this inequality by $\alpha$ and passing to the limit as $\alpha \rightarrow+0$ one gets that the function $\left\|F_{p}(\cdot)-y\right\|$ is directionally differentiable at $x$, and for any $h \in X$ and $y \in Y$ one has

$$
\begin{array}{r}
\left\|F_{p}(\cdot)-y\right\|^{\prime}(x, h)=\omega^{\prime}\left(F_{p}(x)-y, F_{p}^{\prime}(x, h)\right)=\sup _{y^{*} \in \partial \omega\left(F_{p}(x)-y\right)}\left\langle y^{*}, F_{p}^{\prime}(x, h)\right\rangle \\
=\sup _{y^{*} \in \partial \omega\left(F_{p}(x)-y\right)}\left(\max _{v^{*} \in \underline{\partial}_{x} F\left(x, p ; y^{*}\right)}\left\langle v^{*}, h\right\rangle+\min _{w^{*} \in \bar{\partial}_{x} F\left(x, p ; y^{*}\right)}\left\langle w^{*}, h\right\rangle\right) \\
\leq \sup _{y^{*} \in \partial \omega\left(F_{p}(x)-y\right)} \max _{v^{*} \in\left[\mathscr{D}_{x} F\left(x, p ; y^{*}\right)\right]^{+}}\left\langle v^{*}, h\right\rangle,
\end{array}
$$

if $F(x, p) \neq y$, while

$$
\left.\| F_{p}(\cdot)-y\right)\left\|^{\prime}(x, h)=\right\| F_{p}^{\prime}(x, h) \| \leq \sup _{y^{*} \in S_{Y^{*}}} \max _{v^{*} \in\left[\mathscr{D}_{x} F\left(x, p ; y^{*}\right)\right]^{+}}\left\langle v^{*}, h\right\rangle,
$$

in the case $F(x, p)=y$, since $\|y\|=\sup _{y^{*} \in S_{Y^{*}}}\left\langle y^{*}, y\right\rangle$.

Now we can utilize q.d.-MFCQ and the outer semicontinuity of the quasidifferential mappings to prove the inequality $\left|\nabla \psi_{(y, z, p)}\right|(x)>K^{-1}$. Let $\varkappa>0$ be any number smaller than the infimum in (7). From assumption 3 the fact that the set $D(y)$ is convex, and the separation theorem it follows that for any $y \in S_{Y}$ there exists $h_{y}$ with $\left\|h_{y}\right\|=1$ such that $\left\langle v^{*}, h_{y}\right\rangle \leq-\varkappa$ for all $v^{*} \in D(y)$. With the use of the second condition in q.d.-MFCQ one obtains that $\left\langle v^{*}, h_{y}+t \bar{h}\right\rangle \leq-\varkappa$ for all $v^{*} \in D(y)$ and $t \geq 0$, where the vector $\bar{h}$ is from q.d.-MFCQ. Hence applying the fact that the mapping $(x, p) \mapsto\left[\mathscr{D}_{x} F\left(x, p ; y^{*}\right)\right]^{+}$ is o.s.c. at $(\bar{x}, \bar{p})$ uniformly with respect to $y^{*} \in S_{Y^{*}}$, one gets that for any $t \geq 0$ there exists $r_{1}(t) \in(0, \bar{r})$ such that for any $y \in S_{Y}$ one has

$$
\left\langle v^{*}, h_{y}+t \bar{h}\right\rangle \leq-\frac{\varkappa}{2} \quad \forall v^{*} \in\left[\mathscr{D}_{x} F\left(x, p ; y^{*}\right)\right]^{+} \quad \forall y^{*} \in \partial\|\cdot\|(y)
$$

for all $(x, p) \in B\left(\bar{x}, r_{1}(t)\right) \times B\left(\bar{p}, r_{1}(t)\right)$. Furthermore, from the second condition in q.d.-MFCQ and assumption 2 it follows that for any $t \geq 0$ there exists $r_{2}(t) \in(0, \bar{r})$ such that

$$
\left\langle v^{*}, t \bar{h}\right\rangle \leq \frac{\varkappa}{4} \quad \forall v^{*} \in\left[\mathscr{D}_{x} F\left(x, p ; y^{*}\right)\right]^{+} \quad \forall y^{*} \in S_{y^{*}}
$$

for all $(x, p) \in B\left(\bar{x}, r_{2}(t)\right) \times B\left(\bar{p}, r_{2}(t)\right)$.

Applying the second condition in q.d.-MFCQ, and the facts that $\left\|h_{y}\right\|=1$ for any $y \in S_{Y}$ and the sets $\left[\mathscr{D}_{x} g_{i}(\bar{x}, \bar{p})\right]^{+}$are obviously weak* compact (and 
thus bounded) one can find $t_{0}>0$ such that $\left\langle v^{*}, h_{y}+t_{0} \bar{h}\right\rangle \leq-\varkappa$ for all $v^{*} \in\left[\mathscr{D}_{x} g_{i}(\bar{x}, \bar{p})\right]^{+}, i \in I(\bar{x}, \bar{p})$, and $y \in S_{Y}$. Hence with the use of the outer semicontinuity of the mappings $\mathscr{D}_{x} g_{i}(\cdot)$ at $(\bar{x}, \bar{p})$ one obtains that there exists $r_{3} \in(0, \bar{r})$ such that

$$
\left\langle v^{*}, h_{y}+t_{0} \bar{h}\right\rangle \leq-\frac{\varkappa}{2} \quad \forall v^{*} \in\left[\mathscr{D}_{x} g_{i}(x, p)\right]^{+} \forall i \in I(\bar{x}, \bar{p}) \forall y \in S_{Y} .
$$

for all $(x, p) \in B\left(\bar{x}, r_{3}\right) \times B\left(\bar{p}, r_{3}\right)$. Finally, since $g_{i}$ are continuous, there exists $r_{4} \in(0, \bar{r})$ and $\varepsilon>0$ such that $g_{i}(x, p)<-\varepsilon$ for any $(x, p) \in B\left(\bar{x}, r_{4}\right) \times B\left(\bar{p}, r_{4}\right)$ and $i \notin I(\bar{x}, \bar{p})$.

Define $r=\min \left\{r_{1}\left(t_{0}\right), r_{2}\left(t_{0}\right), r_{3}, r_{4}, \varepsilon / 2\right\}$, and fix any $(x, p) \in B(\bar{x}, r) \times$ $B(\bar{p}, r)$ and $(y, z) \in B\left(\left(\mathbb{O}_{Y}, \mathbf{0}_{m}\right), r\right)$ such that $(y, z) \notin \Phi_{p}(x)$. Note that $g_{i}(x, p)-$ $z_{i}<0$ for any $i \notin I(\bar{x}, \bar{p})$, since $r \leq \min \left\{r_{4}, \varepsilon / 2\right\}$, which implies that $g_{i}(\cdot)-z_{i}<0$ in a neighbourhood of $(x, p)$ for any such $i$. Hence

$$
d\left((y, z), \Phi_{q}(\xi)\right)=\|F(\xi, q)-y\|+\sum_{i \in I(\bar{x}, \bar{p})} \max \left\{g_{i}(\xi, q)-z_{i}, 0\right\}
$$

for any $(\xi, q)$ in a neighbourhood of $(x, p)$, i.e. the indices $i \notin I(\bar{x}, \bar{p})$ can be discarded from consideration. Observe also that

$$
\max \left\{g_{i}(\cdot, p)-z_{i}, 0\right\}^{\prime}(x, h)= \begin{cases}{\left[g_{i}(\cdot, p)\right]^{\prime}(x, h),} & \text { if } g_{i}(x, p)>z_{i}, \\ \max \left\{\left[g_{i}(\cdot, p)\right]^{\prime}(x, h), 0\right\}, & \text { if } g_{i}(x, p)=z_{i}, \\ 0, & \text { if } g_{i}(x, p)<z_{i},\end{cases}
$$

and $\left[g_{i}(\cdot, p)\right]^{\prime}(x, h) \leq \max _{v^{*} \in\left[\mathscr{D}_{x} g_{i}(x, p)\right]^{+}}\left\langle v^{*}, h\right\rangle$ for any $h \in X$.

If $F(x, p) \neq y$, then with the use of (12), (14), (16), and (17) one obtains that

$$
\psi_{(y, z, p)}^{\prime}(x, \eta)=\|F(\cdot, p)-y\|^{\prime}(x, \eta)+\sum_{i \in I(\bar{x}, \bar{p})} \max \left\{g_{i}(\cdot, p)-z_{i}, 0\right\}^{\prime}(x, \eta) \leq-\frac{\varkappa}{2}
$$

where $\eta=h_{w}+t_{0} \bar{h}$ and $w=(F(x, p)-y) /\|F(x, p)-y\|$ (here we used the fact that $\partial\|\cdot\|(F(x, p)-y)=\partial\|\cdot\|(w))$. Note that $\|\eta\| \leq 1+t_{0}\|\bar{h}\|$, since $\left\|h_{w}\right\|=1$.

On the other hand, if $F(x, p)=y$, then there exists $k \in I(\bar{x}, \bar{p})$ such that $g_{k}(x, p)>z_{k}$. Consequently, applying (13), (15), (16), and (17) one gets that

$$
\begin{aligned}
\psi_{(y, z, p)}^{\prime}(x, \eta) & =\|F(\cdot, p)-y\|^{\prime}(x, \eta)+\max \left\{g_{k}(\cdot, p)-z_{k}, 0\right\}^{\prime}(x, \eta) \\
& +\sum_{i \in I(\bar{x}, \bar{p}) \backslash\{k\}} \max \left\{g_{i}(\cdot, p)-z_{i}, 0\right\}^{\prime}(x, \eta) \leq \frac{\varkappa}{4}-\frac{\varkappa}{2}=-\frac{\varkappa}{4},
\end{aligned}
$$

where $\eta=t_{0} \bar{h}$. Thus, for any $(x, p) \in B(\bar{x}, r) \times B(\bar{p}, r)$ and $(y, z) \in$ $B\left(\left(\mathbb{O}_{Y}, \mathbf{0}_{m}\right), r\right)$ such that $(y, z) \notin \Phi_{p}(x)$ one has

$$
\left|\nabla \psi_{(y, z, p)}\right|(x) \geq-\psi_{(y, z, p)}^{\prime}\left(x, \frac{\eta}{\|\eta\|}\right) \geq \frac{\varkappa}{4\left(1+t_{0}\|\bar{h}\|\right)},
$$

and the proof is complete.

Remark 6. Let $F$ be as in Proposition 1 and $X=\mathbb{R}^{n}$. In this case one can reformulate the sufficient conditions for the metric regularity of the mapping $F$ 
from the theorem above in a different way. Namely, let the set $\underline{\partial}_{x} F(\bar{x}, \bar{p})$ consists of all $l \times n$ matrices whose $j$-th row is a vector from $\underline{\partial}_{x} f_{j}(\bar{x}, \bar{p})$. The set $\bar{\partial}_{x} F(\bar{x}, \bar{p})$ is defined in a similar way. Then the pair $\mathscr{D}_{x} F(\bar{x}, \bar{p})=\left[\underline{\partial}_{x} F(\bar{x}, \bar{p}), \bar{\partial}_{x} F(\bar{x}, \bar{p})\right]$ is, in fact, a quasidifferential of the mapping $F(\cdot, \bar{p})$ at $\bar{x}$ (see [10, Appendix III]). From Theorem 2 it follows that for the mapping $F(\cdot, p)$ to be metrically regular near $(\bar{x}, F(\bar{x}, p))$ with the norm of metric regularity not exceeding some $K>0$ for all $p$ in a neighbourhood of $\bar{p}$ it is sufficient that $l \leq n$, and all matrices from the set $\left[\mathscr{D}_{x} F(\bar{x}, \bar{p})\right]^{+}=\underline{\partial}_{x} F(\bar{x}, \bar{p})+\bar{\partial}_{x} F(\bar{x}, \bar{p})$ have full rank. Note that a similar condition on the set $\left[\mathscr{D}_{x} F(\bar{x}, \bar{p})\right]^{+}$was introduced by Demyanov in [7] for the analysis of nonsmooth implicit functions and a nonsmooth Newton method for codifferentiable vector-valued functions.

Remark 7 . It should be noted that in the case when $X=\mathbb{R}^{n}$ and $Y=\mathbb{R}^{l}$, Theorem 2 is, in essence, reduced to the sufficient conditions for metric regularity in terms of the Clarke subdifferential [1,4. Indeed, if a function $f: X \rightarrow \mathbb{R}$ is quasidifferentiable at a point $x$, then, as it easy to see,

$$
\min _{v^{*} \in[\mathscr{D} f(x)]^{+}}\left\langle v^{*}, h\right\rangle \leq f^{\prime}(x, h) \leq \max _{v^{*} \in[\mathscr{D} f(x)]^{+}}\left\langle v^{*}, h\right\rangle \quad \forall h \in X,
$$

i.e. the quasidifferential sum $[\mathscr{D} f(x)]^{+}$is a convexificator of $f$ at $x$ (see $[8,9,25]$ ). With the use of the separation theorem and the inequalities above one can easily check that if $f$ is Gâteaux differentiable at $x$, then $f^{\prime}(x) \in[\mathscr{D} f(x)]^{+}$regardless of the choice of quasidifferential. Consequently, if $X=\mathbb{R}^{n}, f$ is Lipschitz continuous and quasidifferentiable near $x$, and a quasidifferential mapping $\mathscr{D} f$ is o.s.c. at $x$, then $\partial_{C l} f(x) \subseteq[\mathscr{D} f(x)]^{+}$, where $\partial_{C l} f(x)$ is the Clarke subdifferential of $f$ at $x[5]$.

With the use of [14, Corollary 2] one can verify that under the assumptions of Theorem 2 the functions $F(\cdot, p)$ and $g_{i}(\cdot, p)$ are Lipschitz continuous near $\bar{x}$ with the same Lipschitz constant for all $p$ in a neighbourhood of $\bar{p}$, provided $F$ has the same form as in Proposition [1. Therefore, if $X=\mathbb{R}^{n}$, then $\partial_{C l} g_{i}(\cdot, \bar{p})(\bar{x}) \subseteq$ $\left[\mathscr{D}_{x} g_{i}(\bar{x}, \bar{p})\right]^{+}$, and the same inclusion holds true for $f_{j}(x, p)$. Thus, if $X=\mathbb{R}^{n}$ and $Y=\mathbb{R}^{l}$, then Theorem 2 is a corollary to the sufficient conditions for metric regularity in terms of the Clarke subdifferential [1, Theorem 1.1] (see also 4]). On the other hand, if either $X$ or $Y$ is infinite dimensional, then Theorem 2 does not follow from the main results of [1,4].

Let us also point out that Theorem 2 can be easily extended to the case when instead of quasidifferential sums one uses o.s.c. convexificator mappings. However, since the Clarke subdifferential is the smallest o.s.c. convexificator mapping, in the finite dimensional case this result is a corollary to [1, Theorem 1.1] as well.

Let us give an example illustrating Theorem 2 and Remark 6

Example 2. Let $X=Y=\mathbb{R}^{2}$ and $P=\mathbb{R}$. Consider the following system of equations:

$$
\left\{\begin{array}{l}
\max \left\{2 x_{1}, x_{1}\right\}-\left|\sin \left(p x_{2}\right)\right|=y_{1}, \\
\sin \left(p\left(x_{1}+x_{2}\right)\right)+\min \left\{x_{2}, 2 x_{2}\right\}=y_{2} .
\end{array}\right.
$$

Define $f_{1}(x, p)=\max \left\{3 x_{1}, x_{1}\right\}-\left|\sin \left(p x_{2}\right)\right|$ and $f_{2}(x, p)=\sin \left(p\left(x_{1}+x_{2}\right)\right)+$ $\min \left\{x_{2}, 2 x_{2}\right\}$. Let us utilize Theorem 2 to find the values of the parameter $p$ for which the mapping $x \mapsto F(x, p)=\left(f_{1}(x, p), f_{2}(x, p)\right)^{T}$ is metrically regular near the point $\left(\mathbf{0}_{2}, \mathbf{0}_{2}\right)$. 
The functions $f_{1}(x, p)$ and $f_{2}(x, p)$ are quasidifferentiable. With the use of basic rules of quasidifferential calculus [10, Section III.2] one obtains that

$$
\begin{aligned}
& \underline{\partial}_{x} f_{1}(x, p)= \begin{cases}\left\{(2,0)^{T}\right\}, & \text { if } x_{1}>0, \\
\operatorname{co}\left\{(1,0)^{T},(2,0)^{T}\right\}, & \text { if } x_{1}=0, \\
\left\{(1,0)^{T}\right\}, & \text { if } x_{1}<0,\end{cases} \\
& \left.\bar{\partial}_{x} f_{1}(x, p)=\left\{\begin{array}{ll}
\left(-p \cos \left(p x_{2}\right) \operatorname{Sign}\left(\sin \left(p x_{2}\right)\right)\right.
\end{array}\right)\right\}, \\
& \left.\underline{\partial}_{x} f_{2}(x, p)=\left\{\begin{array}{ll}
p \cos \left(p\left(x_{1}+x_{2}\right)\right) \\
p \cos \left(p\left(x_{1}+x_{2}\right)\right)
\end{array}\right)\right\}, \\
& \bar{\partial}_{x} f_{2}(x, p)= \begin{cases}\left\{(0,1)^{T}\right\}, & \text { if } x_{2}>0, \\
\operatorname{co}\left\{(0,1)^{T},(0,2)^{T}\right\}, & \text { if } x_{2}=0, \\
\{(0,2)\}, & \text { if } x_{2}<0 .\end{cases}
\end{aligned}
$$

It is readily seen that the quasidifferential mappings $(x, p) \mapsto \mathscr{D}_{x} f_{1}(x, p)$ and $(x, p) \mapsto \mathscr{D}_{x} f_{2}(x, p)$ are outer semicontinuous.

Let us verify whether q.d.-MFCQ holds at the point $\left(\mathbf{0}_{2}, p\right)$. Following Remark 6 introduce the quasidifferential $\mathscr{D}_{x} F\left(\mathbf{0}_{2}, p\right)=\left[\underline{\partial}_{x} F\left(\mathbf{0}_{2}, p\right), \bar{\partial}_{x} F\left(\mathbf{0}_{2}, p\right)\right]$,

$$
\begin{aligned}
& \underline{\partial}_{x} F\left(\mathbf{0}_{2}, p\right)=\left\{\left(\begin{array}{cc}
t & 0 \\
p & p
\end{array}\right) \mid t \in[1,2]\right\}, \\
& \bar{\partial}_{x} F\left(\mathbf{0}_{2}, p\right)=\left\{\left(\begin{array}{cc}
0 & p t \\
0 & s
\end{array}\right) \mid t \in[-1,1], s \in[1,2]\right\},
\end{aligned}
$$

of the map $x \mapsto F(x, p)$ at the point $x=\mathbf{0}_{2}$. The first row of the set $\underline{\partial}_{x} F\left(\mathbf{0}_{2}, p\right)$ corresponds to $\underline{\partial}_{x} f_{1}\left(\mathbf{0}_{2}, p\right)$, while the second row corresponds to $\underline{\partial}_{x} f_{2}\left(\mathbf{0}_{2}, p\right)$. The set $\bar{\partial}_{x} F\left(\mathbf{0}_{2}, p\right)$ is defined in the same way.

The quasidifferential sum of the map $x \mapsto F(x, p)$ at $x=\mathbf{0}_{2}$ has the form

$$
\left[\mathscr{D}_{x} F\left(\mathbf{0}_{2}, p\right)\right]^{+}=\left\{\left(\begin{array}{cc}
t & p s \\
p & p+r
\end{array}\right) \mid t \in[1,2], s \in[-1,1], r \in[1,2]\right\}
$$

Our aim is to find such $p \in \mathbb{R}$ that all matrices from the set $\left[\mathscr{D}_{x} F\left(\mathbf{0}_{2}, p\right)\right]^{+}$ are nondegenerate. The determinants of the matrices from $\left[\mathscr{D}_{x} F\left(\mathbf{0}_{2}, p\right)\right]^{+}$take values in the set

$$
\operatorname{co}\{1,4\}+\operatorname{co}\{p, 2 p\}+\operatorname{co}\left\{-p^{2}, p^{2}\right\}
$$

Hence taking into accoun the fact that the determinant of $\left(\begin{array}{ll}1 & -p \\ p & p+1\end{array}\right) \in$ $\left[\mathscr{D}_{x} F\left(\mathbf{0}_{2}, p\right)\right]^{+}$is equal to $p^{2}+p+1$ and positive for all $p$ one obtains that $\operatorname{det} A \neq 0$ for any $A \in\left[\mathscr{D}_{x} F\left(\mathbf{0}_{2}, p\right)\right]^{+}$iff the following inequalities hold true:

$$
p^{2}+2 p+1>0, \quad-p^{2}+p+1>0, \quad-p^{2}+2 p+1>0 .
$$

Solving these inequalities one obtains that q.d.-MFCQ holds at the point $\left(\mathbf{0}_{2}, p\right)$ iff $p \in(1-\sqrt{2},(1+\sqrt{5}) / 2)$. Consequently, by Theorem 2 one can conclude that for any $\bar{p} \in(1-\sqrt{2},(1+\sqrt{5}) / 2)$ there exist $K>0$ and $r>0$ such that

$$
d\left(x,\left(F_{p}\right)^{-1}(y)\right) \leq K\|y-F(x, p)\|
$$

for all $x, y \in B\left(\mathbf{0}_{2}, r\right)$ and any $p \in(\bar{p}-r, \bar{p}+r)$, which in particular implies that for any such $y$ and $p$ there exists a solution $x(y, p)$ of system (18). 
As the following simple example shows q.d.-MFCQ, unlike MFCQ in the smooth case, is not necessary for the metric regularity of a multifunction associated with a system of quasidifferentiable equality and inequality constraints.

Example 3. Let $X=\mathbb{R}^{2}, Y=\mathbb{R}, F(x)=\left|x_{1}\right|-\left|x_{2}\right|$, and suppose that there are no inequality constraints. Let us check whether q.d.-MFCQ holds at the point $\bar{x}=\mathbf{0}_{2}$. Indeed, the function $F$ is quasidifferentiable, and one can define

$$
\underline{\partial} F(x)=\left\{\left(\begin{array}{c}
\operatorname{Sign}\left(x_{1}\right) \\
0
\end{array}\right)\right\}, \quad \bar{\partial} F(x)=\left\{\left(\begin{array}{c}
0 \\
-\operatorname{Sign}\left(x_{2}\right)
\end{array}\right)\right\} .
$$

Clearly, the multifunctions $\underline{\partial} F(\cdot)$ and $\bar{\partial} F(\cdot)$ are outer semicontinuous. Observe that $[\mathscr{D} F(\bar{x})]^{+}=\left\{x \in \mathbb{R}^{2} \mid \max \left\{\left|x_{1}\right|,\left|x_{2}\right|\right\} \leq 1\right\}$, and q.d.-MFCQ is not satisfied at the origin, since $\mathbf{0}_{2} \in[\mathscr{D} F(\bar{x})]^{+}$, despite the fact that the function $F$ is metrically regular near the point $(\bar{x}, 0)$ (see Remark 4).

It should be noted that in the finite dimensional case q.d.-MFCQ imposes some implicit assumptions on the dimension of the space $X$. For example, if for the system

$$
f_{1}(x, p)=y, \quad g_{1}(x, p) \leq 0
$$

the quasidifferential sum $\left[\mathscr{D}_{x} f_{1}(\bar{x}, \bar{p})\right]^{+}$contains at least two linearly independent vectors, then $\operatorname{dim}\left(\operatorname{span}\left[\mathscr{D}_{x} f_{1}(\bar{x}, \bar{p})\right]^{+}\right) \geq 2$ and for q.d.-MFCQ to hold true at $(\bar{x}, \bar{p})$ it is necessary that $\operatorname{dim} X \geq 3$ (see Remark 5 ). The following example highlights this drawback of q.d.-MFCQ.

Example 4. Let $X=\mathbb{R}^{2}, Y=\mathbb{R}$, and $m=1$. Consider the following system:

$$
f(x)=\left|x_{1}\right|-x_{2}=y, \quad g(x)=x_{1} \leq z .
$$

Our aim is to check whether the multifunction $\Phi(x)=\{f(x)\} \times[g(x),+\infty)$ associated with this system is metrically regular near the point $(\bar{x},(0,0))$ with $\bar{x}=\mathbf{0}_{2}$.

Both functions $f$ and $g$ are obviously quasidifferentiable. One can define

$$
\begin{aligned}
& \underline{\partial} f(x)=\left\{\left(\begin{array}{c}
\operatorname{Sign}\left(x_{1}\right) \\
-1
\end{array}\right)\right\}, \quad \bar{\partial} f(x)=\left\{\mathbf{0}_{2}\right\}, \\
& \underline{\partial} g(x)=\left\{\left(\begin{array}{l}
1 \\
0
\end{array}\right)\right\}, \quad \bar{\partial} g(x)=\left\{\mathbf{0}_{2}\right\} .
\end{aligned}
$$

Clearly, the mappings $\mathscr{D} f(\cdot)$ and $\mathscr{D} g(\cdot)$ are outer semicontinuous. Observe that

$$
[\mathscr{D} f(\bar{x})]^{+}=\operatorname{co}\left\{\left(\begin{array}{c}
1 \\
-1
\end{array}\right),\left(\begin{array}{l}
-1 \\
-1
\end{array}\right)\right\}, \quad[\mathscr{D} g(\bar{x})]^{+}=\left\{\left(\begin{array}{l}
1 \\
0
\end{array}\right)\right\} .
$$

Hence $\operatorname{span}[\mathscr{D} f(x)]^{+}=\mathbb{R}^{2}$, which implies that q.d.-MFCQ does not hold at $\bar{x}$, and Theorem 2 cannot be applied. Therefore we utilize Theorem 1 to check whether the multifunction $\Phi$ is metrically regular near the point $(\bar{x},(0,0))$.

Note that

$$
\psi_{(y, z)}(x)=d((y, z), \Phi(x))=|y-| x_{1}\left|+x_{2}\right|+\max \left\{0, x_{1}-z\right\} .
$$


Define $\psi_{y}^{1}(x)=|y-| x_{1}\left|+x_{2}\right|$ and $\psi_{z}^{2}(x)=\max \left\{0, x_{1}-z\right\}$. The functions $\psi_{(y, z)}(\cdot), \psi_{y}^{1}(\cdot)$ and $\psi_{z}^{2}(\cdot)$ are quasidifferentiable for all $y, z \in \mathbb{R}$. Applying basic rules of quasidifferential calculus [10, Section III.2] one obtains that

$$
\begin{aligned}
& \underline{\partial} \psi_{y}^{1}(x)=\left\{\mathbf{0}_{2}\right\}, \quad \bar{\partial} \psi_{y}^{1}(x)=\left\{\left(\begin{array}{c}
-\operatorname{Sign}\left(x_{1}\right) \\
1
\end{array}\right)\right\}, \quad \text { if } y>f(x), \\
& \underline{\partial} \psi_{y}^{1}(x)=\operatorname{co}\left\{\left(\begin{array}{c}
0 \\
0
\end{array}\right),\left(\begin{array}{c}
2 \operatorname{Sign}\left(x_{1}\right) \\
-2
\end{array}\right)\right\}, \bar{\partial} \psi_{y}^{1}(x)=\left\{\left(\begin{array}{c}
-\operatorname{Sign}\left(x_{1}\right) \\
1
\end{array}\right)\right\}, \text { if } y=f(x), \\
& \underline{\partial} \psi_{y}^{1}(x)=\left\{\left(\begin{array}{c}
\operatorname{Sign}\left(x_{1}\right) \\
-1
\end{array}\right)\right\}, \quad \bar{\partial} \psi_{y}^{1}(x)=\left\{\mathbf{0}_{2}\right\}, \quad \text { if } y<f(x), \\
& \underline{\partial} \psi_{z}^{2}(x)= \begin{cases}\left\{\mathbf{0}_{2}\right\}, & \text { if } x_{1}<z, \\
\operatorname{co}\left\{(0,0)^{T},(1,0)^{T}\right\}, & \text { if } x_{1}=z, \\
\left\{(1,0)^{T}\right\}, & \text { if } x_{1}>z,\end{cases}
\end{aligned}
$$

Moreover, $\underline{\partial} \psi_{(y, z)}(x)=\underline{\partial} \psi_{y}^{1}(x)+\underline{\partial} \psi_{z}^{2}(x)$ and $\bar{\partial} \psi_{(y, z)}(x)=\bar{\partial} \psi_{y}^{1}(x)+\bar{\partial} \psi_{z}^{2}(x)$.

Fix any $x \in \mathbb{R}^{2}$ and $y, z \in \mathbb{R}$ such that $(y, z) \notin \Phi(x)$, and suppose that the space $X$ is equipped with the Euclidean norm. The following three cases are possible.

1. If $y>f(x)$, then for any $t \in \operatorname{Sign}\left(x_{1}\right)$ one has $w^{*}=(-t, 1)^{T} \in \bar{\partial} \psi_{(y, z)}(x)$ and $d\left(\mathbf{0}_{2}, \underline{\partial} \psi_{(y, z)}(x)+w^{*}\right) \geq 1$, since any $v^{*} \in \underline{\partial} \psi_{(y, z)}(x)+w^{*}$ has the form $(s, 1)^{T}$ for some $s \in \mathbb{R}$.

2. If $y<f(x)$, then for $w^{*}=\mathbf{0}_{2} \in \bar{\partial} \psi_{(y, z)}(x)$ one has $d\left(\mathbf{0}_{2}, \underline{\partial} \psi_{(y, z)}(x)+w^{*}\right) \geq$ 1 , since any $v^{*} \in \underline{\partial} \psi_{(y, z)}(x)+w^{*}$ has the form $(s,-1)^{T}$ for some $s \in \mathbb{R}$.

3. If $y=f(x)$, then $x_{1}>z$ due to the fact that $(y, z) \notin \Phi(x)$. Define $w^{*}=\left(-\operatorname{sign}\left(x_{1}\right), 1\right) \in \bar{\partial} \psi_{(y, z)}(x)$, if $x_{1} \neq 0$, and $w^{*}=(1,1) \in \bar{\partial} \psi_{(y, z)}(x)$, if $x_{1}=0$. Then one can verify that $d\left(\mathbf{0}_{2}, \underline{\partial} \psi_{(y, z)}(x)+w^{*}\right)=\sqrt{2} / 2$.

Thus, for any $x \in \mathbb{R}^{2}$ and $y, z \in \mathbb{R},(y, z) \notin \Phi(x)$, there exists $w^{*} \in \bar{\partial} \psi_{(y, z)}(x)$ such that $d\left(\mathbf{0}_{2}, \underline{\partial} \psi_{(y, z)}(x)+w^{*}\right) \geq \sqrt{2} / 2$. Therefore, the multifunction $\Phi$ is metrically regular near the point $(\bar{x},(0,0))$ with the norm of metric regularity not exceeding $\sqrt{2} / 2$ by Theorem 1 .

\section{Optimality Conditions}

Let us utilize q.d.-MFCQ as a new constraint qualification for quasidifferential programming problems with equality and inequality constraints to obtain necessary optimality conditions for these problems. To this end, consider the following optimization problem:

$$
\min u(x) \text { subject to } f_{j}(x)=0, \quad j \in J, \quad g_{i}(x) \leq 0, \quad i \in I .
$$

Here $u, f_{j}, g_{i}: X \rightarrow \mathbb{R}$ are given functions, $J=\{1, \ldots, l\}$, and $I=\{1, \ldots, m\}$. Our aim is to obtain optimality conditions for the problem $(\mathcal{P})$ via exact penalty function approach.

Define $\varphi(x)=\sum_{j=1}^{l}\left|f_{j}(x)\right|+\sum_{i=1}^{m} \max \left\{g_{i}(x), 0\right\}$, and denote the $\ell_{1}$ penalty function for the problem $(\mathcal{P})$ by $\Psi_{c}(x)=u(x)+c \varphi(x)$, where $c \geq 0$ is the penalty 
parameter. Note that if the functions $u, f_{j}$, and $g_{i}$ are quasidifferentiable, then this penalty function is quasidifferentiable as well (see [10]).

Let $\Omega$ be the feasible region of the problem $(\mathcal{P})$, and $\bar{x}$ be a locally optimal solution of this problem. Observe that $x \in \Omega$ iff $\varphi(x)=0$. Recall also that if $u$ is Lipschitz continuous near $\bar{x}$, and the penalty term $\varphi$ has a local error bound at $\bar{x}$, i.e. there exists $\tau>0$ such that $\varphi(x) \geq \tau d(x, \Omega)$ for any $x$ in a neighbourhood of $\bar{x}$, then the penalty function $\Psi_{c}$ is locally exact at $\bar{x}$, i.e. there exist a neighbourhood $U$ of $\bar{x}$ and $c^{*} \geq 0$ such that

$$
\Psi_{c}(x) \geq \Psi_{c}(\bar{x}) \quad \forall x \in U \quad \forall c \geq c^{*},
$$

(see, e.g. [13, Theorem 2.4 and Proposition 2.7]). If $\Psi_{c}$ is locally exact at $\bar{x}$, then by definition $\bar{x}$ is a point of unconstrained local minimum of $\Psi_{c}$ for any sufficiently large $c \geq 0$. In this case one can apply standard necessary conditions for a minimum in terms of quasidifferentials [10] to $\Psi_{c}$ to obtain necessary optimality conditions for the problem $(\mathcal{P})$.

Theorem 3. Let the following assumptions be valid:

1. $\bar{x}$ is a locally optimal solution of the problem $(\mathcal{P})$;

2. $u$ is quasidifferentiable at $\bar{x}$ and Lipschitz continuous near this point;

3. $f_{j}, j \in J$, and $g_{i}, i \in I$, are quasidifferentiable in a neighbourhood of $\bar{x}$, and there exist quasidifferential mappings $\mathscr{D} f_{j}(\cdot), j \in J$, and $\mathscr{D} g_{i}(\cdot)$, $i \in I$, defined in a neighbourhood of $\bar{x}$ and o.s.c. at this point;

4. q.d.-MFCQ holds at $\bar{x}$.

Then there exists $c^{*} \geq 0$ such that for any $c \geq c^{*}$ one has

$$
\mathbb{O} \in \underline{\partial} \Psi_{c}(\bar{x})+w^{*} \quad \forall w^{*} \in \bar{\partial} \Psi_{c}(\bar{x}),
$$

where $\mathscr{D} \Psi_{c}(\bar{x})=\left[\underline{\partial} \Psi_{c}(\bar{x}), \bar{\partial} \Psi_{c}(\bar{x})\right]$ is any quasidifferential of $\Psi_{c}$ at $\bar{x}$. Moreover, for any $w_{0}^{*} \in \bar{\partial} u(\bar{x}), v_{j}^{*} \in \underline{\partial} f_{j}(\bar{x}), w_{j}^{*} \in \bar{\partial} f_{j}(\bar{x}), j \in J$, and $z_{i}^{*} \in \bar{\partial} g_{i}(\bar{x}), i \in I$, there exist $\underline{\mu}_{j}, \bar{\mu}_{j}, \lambda_{i} \geq 0$ such that $\lambda_{i} g_{i}(\bar{x})=0$ for all $i \in I$ and

$$
\begin{aligned}
\mathbb{O} \in \underline{\partial} u(\bar{x})+w_{0}^{*} & -\sum_{j=1}^{l} \underline{\mu}_{j}\left(v_{j}^{*}+\bar{\partial} f_{j}(\bar{x})\right) \\
& +\sum_{j=1}^{l} \bar{\mu}_{j}\left(\underline{\partial} f_{j}(\bar{x})+w_{j}^{*}\right)+\sum_{i=1}^{m} \lambda_{i}\left(\underline{\partial} g_{i}(\bar{x})+z_{i}^{*}\right) .
\end{aligned}
$$

In addition, one can choose $\underline{\mu}_{j}, \bar{\mu}_{j}$, and $\lambda_{i}$ in such a way that for all $i \in I$ and $j \in J$ one has $\max \left\{\underline{\mu}_{j}+\bar{\mu}_{j}, \lambda_{i}\right\} \leq c^{*}$, i.e. the multipliers $\underline{\mu}_{j}, \bar{\mu}_{j}$, and $\lambda_{i}$ are bounded for all $w_{0}^{*} \in \bar{\partial} u(\bar{x}), v_{j}^{*} \in \underline{\partial} f_{j}(\bar{x}), w_{j}^{*} \in \bar{\partial} f_{j}(\bar{x}), j \in J$, and $z_{i}^{*} \in \bar{\partial} g_{i}(\bar{x})$, $i \in I$.

Proof. Let us show at first that q.d.-MFCQ guarantees that $\varphi$ has a local error bound. Suppose that $\mathbb{R}^{l}$ is endowed with the Euclidean norm. If q.d.-MFCQ holds at $\bar{x}$, then by Theorem 2 the multifunction $\Phi: X \rightarrow \mathbb{R}^{l} \times \mathbb{R}^{m}, \Phi(x)=$ 
$\prod_{j=1}^{l}\left\{f_{j}(x)\right\} \times \prod_{i=1}^{m}\left[g_{i}(x),+\infty\right)$ is metrically regular near the point $\left(\bar{x},\left(\mathbf{0}_{l}, \mathbf{0}_{m}\right)\right)$. Hence, in particular, there exist $K>0$ and a neighbourhood $U$ of $\bar{x}$ such that

$$
d(x, \Omega)=d\left(x, \Phi^{-1}\left(\mathbf{0}_{l}, \mathbf{0}_{m}\right)\right) \leq K d\left(\left(\mathbf{0}_{l}, \mathbf{0}_{m}\right), \Phi(x)\right) \leq K \varphi(x)
$$

for all $x \in U$, i.e. $\varphi$ has a local error bound at $\bar{x}$.

Now we can turn to the proof of (19). Under the assumptions of the theorem the penalty function $\Psi_{c}$ is locally exact at $\bar{x}$ by [13, Theorem 2.4 and Proposition 2.7]. Thus, there exists $c^{*} \geq 0$ such that for any $c \geq c^{*}$ the point $\bar{x}$ is a local minimizer of $\Psi_{c}$. Consequently, applying the necessary conditions for a minimum in terms of quasidifferentials [10, Theorem V.3.1] to $\Psi_{c}$ one gets that $\mathbb{Q}\left(\mathbb{\partial} \Psi_{c}(\bar{x})+w^{*}\right.$ for all $w^{*} \in \bar{\partial} \Psi_{c}(\bar{x})$, i.e. (19) holds true.

To prove the validity of (20) note that by the necessary condition for a minimum in terms of directional derivative for all $c \geq c^{*}$ and $h \in X$ one has

$$
\Psi_{c}^{\prime}(\bar{x}, h)=u^{\prime}(\bar{x}, h)+c\left(\sum_{j=1}^{l}\left|f_{j}^{\prime}(\bar{x}, h)\right|+\sum_{i \in I(\bar{x})} \max \left\{g_{j}^{\prime}(\bar{x}, h), 0\right\}\right) \geq 0,
$$

where $I(\bar{x})=\left\{i \in I \mid g_{i}(\bar{x})=0\right\}$ (here we used standard calculus rules for directional derivatives; see, e.g. [10, Sect. I.3]). Let $w_{0}^{*}, v_{j}^{*}, w_{j}^{*}$ and $z_{i}^{*}$ be as in the formulation of the theorem. Define $s(C, h)=\sup _{x^{*} \in C}\left\langle x^{*}, h\right\rangle$ for any $C \subset X^{*}$, and

$$
\begin{aligned}
\xi_{c}(h)=s\left(\underline{\partial} u(\bar{x})+w_{0}^{*}, h\right) & +c \sum_{j=1}^{l} \max \left\{s\left(\underline{\partial} f_{j}(\bar{x})+w_{j}^{*}, h\right), s\left(-v_{j}^{*}-\bar{\partial} f_{j}(\bar{x}), h\right)\right\} \\
& +c \sum_{i \in I(\bar{x})} \max \left\{s\left(\underline{\partial} g_{i}(\bar{x})+z_{i}^{*}, h\right), 0\right\} \quad \forall h \in X .
\end{aligned}
$$

Applying the definition of quasidifferential it is easy to see that $\xi_{c}(h) \geq$ $\Psi_{c}^{\prime}(x, h) \geq 0$ for all $c \geq c^{*}$ and $h \in X$. Therefore, $\mathbb{O}$ is a point of global minimum of the function $\xi_{c}$, since $\xi_{c}(\mathbb{O})=0$, which implies that $\mathbb{O} \in \partial \xi_{c}(\mathbb{O})$ for any $c \geq c^{*}$, where $\partial \xi_{c}(\mathbb{O})$ is the subdifferential of $\xi_{c}$ at $\mathbb{O}$ in the sense of convex analysis. Applying standard calculus rules for subdifferentials of convex functions one obtains that

$$
\begin{aligned}
\mathbb{O} \in \partial \xi_{c}(\mathbb{O})=\underline{\partial} u(\bar{x})+w_{0}^{*} & +c \sum_{j=1}^{l} \operatorname{co}\left\{\underline{\partial} f_{j}(\bar{x})+w_{j}^{*},-v_{j}^{*}-\bar{\partial} f_{j}(\bar{x})\right\} \\
& +c \sum_{i \in I(\bar{x})} \operatorname{co}\left\{\underline{\partial} g_{i}(\bar{x})+z_{i}^{*}, \mathbb{O}\right\} .
\end{aligned}
$$

for all $c \geq c^{*}$. Hence for any $c \geq c^{*}$ there exists $\alpha_{j} \in[0,1], j \in J$, and $\beta_{i} \in[0,1]$, $i \in I(\bar{x})$, such that

$$
\begin{aligned}
\mathbb{O} \in \underline{\partial} u(\bar{x})+w_{0}^{*} & +c \sum_{j=1}^{l} \alpha_{j}\left(\underline{\partial} f_{j}(\bar{x})+w_{j}^{*}\right) \\
& -c \sum_{j=1}^{l}\left(1-\alpha_{j}\right)\left(v_{j}^{*}+\bar{\partial} f_{j}(\bar{x})\right)+c \sum_{i=1}^{m} \beta_{i}\left(\underline{\partial} g_{i}(\bar{x})+z_{i}^{*}\right) .
\end{aligned}
$$


Denoting $\underline{\mu}_{j}=c\left(1-\alpha_{j}\right), \bar{\mu}_{j}=c \alpha_{j}, j \in J, \lambda_{i}=c \beta_{i}$ for $i \in I(\bar{x})$, and $\lambda_{i}=0$ for $i \in I \backslash I(\bar{x})$ one obtains that (20) holds true. Note finally that setting $c=c^{*}$ one gets the required bound on multipliers.

Remark 8. Note that in the theorem above instead of q.d.-MFCQ it is sufficient to suppose that the penalty term $\varphi$ has a local error bound at $\bar{x}$.

Remark 9. Optimality conditions similar to but weaker than (19) were obtained in 34,35 in the finite dimensional case under a different constraint qualification that involves some assumptions on so-called contact points of the sets $\underline{\partial} f_{j}(\bar{x})$ and $\bar{\partial} f_{j}(\bar{x})$, i.e. such points $v^{*}$ of a convex set $C \subset X^{*}$ that $s(C, h)=\left\langle v^{*}, h\right\rangle$ for a given direction $h$. Note that one has to compute contact points of the sets $\underline{\partial} f_{j}(\bar{x})$ and $\bar{\partial} f_{j}(\bar{x})$ for all feasible directions in order to check the validity of the constraint qualification from [34,35, which is impossible in nontrivial cases. In contrast, q.d.-MFCQ is formulated in terms of problem data directly. In turn, optimality conditions similar to but weaker than (20) were derived in 33 under yet another constraint qualification in the case when $X$ is finite dimensional, there are no inequality constraints, and there is only one equality constraint. Furthermore, note that sufficient conditions for the validity of this constraint qualification [33, Theorem 2] coincide with q.d.-MFCQ with $I=\emptyset$ and $l=1$.

At first glance optimality condition (19) might seem sharper than condition (20). Let us show that these conditions are in fact equivalent and independent of the choice of quasidifferentials (cf. [28, 29]).

Proposition 2. Let the functions $u, f_{j}, j \in J$, and $g_{i}, i \in I$, be quasidifferentiable at a feasible point $\bar{x}$ of the problem $(\mathcal{P})$. Then (19) is satisfied for some $c \geq 0$ if and only if for any $w_{0}^{*} \in \bar{\partial} u(\bar{x}), v_{j}^{*} \in \underline{\partial} f_{j}(\bar{x}), w_{j}^{*} \in \bar{\partial} f_{j}(\bar{x}), j \in J$, and $z_{i}^{*} \in \bar{\partial} g_{i}(\bar{x}), i \in I$, there exist $\underline{\mu}_{j}, \bar{\mu}_{j}, \lambda_{i} \geq 0$ such that (20) holds true, and for all $i \in I$ and $j \in J$ one has $\lambda_{i} g_{i}(\bar{x})=0$ and $\max \left\{\underline{\mu}_{j}+\bar{\mu}_{j}, \lambda_{i}\right\} \leq c$. Furthermore, both these conditions are independent of the choice of corresponding quasidifferentials.

Proof. From the definition of quasidifferential it follows that

$$
\Psi_{c}^{\prime}(\bar{x}, h)=\min _{w^{*} \in \bar{\partial} \Psi_{c}(\bar{x})} \max _{v^{*} \in \underline{\partial} \Psi_{c}(\bar{x})+w^{*}}\left\langle v^{*}, h\right\rangle \quad \forall h \in X,
$$

which implies that (19) is satisfied for some $c \geq 0$ iff $\Psi_{c}^{\prime}(\bar{x}, h) \geq 0$ for all $h \in X$. The latter condition is obviously independent of the choise of quasidifferential. Therefore optimality condition (19) is independent of the choice of a quasidifferential of $\Psi_{c}$ as well.

Let us now show that optimality conditions (19) and (20) are equivalent. Indeed, let (19) be valid for some quasidifferential of $\Psi_{c}$ at $\bar{x}$ and $c \geq 0$. Then $\Psi_{c}^{\prime}(x, h) \geq 0$ for all $h \in X$. Hence arguing in the same way as in the proof of Theorem 3 one obtains that for any $w_{0}^{*} \in \bar{\partial} u(\bar{x}), v_{j}^{*} \in \underline{\partial} f_{j}(\bar{x}), w_{j}^{*} \in \bar{\partial} f_{j}(\bar{x})$, $j \in J$, and $z_{i}^{*} \in \bar{\partial} g_{i}(\bar{x}), i \in I$, there exist $\underline{\mu}_{j}, \bar{\mu}_{j}, \lambda_{i} \geq 0$ such that (20) holds true, and for all $i \in I, j \in J$ one has $\lambda_{i} g_{i}(\bar{x})=0$ and $\max \left\{\underline{\mu}_{j}+\bar{\mu}_{j}, \lambda_{i}\right\} \leq c$. Note that the implication (19) $\Longrightarrow$ (20) is valid for any quasidifferentials of the functions $u, f_{i}$, and $g_{j}$.

Let us prove the converse implication. Fix any quasidifferentials of the functions $u, f_{i}$, and $g_{j}$, and suppose that there exists $c_{0} \geq 0$ such that for any 
$w_{0}^{*} \in \bar{\partial} u(\bar{x}), v_{j}^{*} \in \underline{\partial} f_{j}(\bar{x}), w_{j}^{*} \in \bar{\partial} f_{j}(\bar{x}), j \in J$, and $z_{i}^{*} \in \bar{\partial} g_{i}(\bar{x}), i \in I$, there exist $\underline{\mu}_{j}, \bar{\mu}_{j}, \lambda_{i} \geq 0$ such that (20) holds true, and for all $i \in I, j \in J$ one has $\lambda_{i} g_{i}(\bar{x})=0$ and $\max \left\{\underline{\mu}_{j}+\bar{\mu}_{j}, \lambda_{i}\right\} \leq c_{0}$.

Arguing by reductio ad absurdum suppose that (19) does not hold true for $c=c_{0}$. Then there exists $h_{0} \in X$ such that $\Psi_{c_{0}}^{\prime}\left(\bar{x}, h_{0}\right)<0$. Applying standard calculus rules for directional derivatives (see, e.g. [10, Sect. I.3]) one obtains that

$$
\begin{aligned}
\Psi_{c_{0}}^{\prime}\left(\bar{x}, h_{0}\right)=u^{\prime}\left(\bar{x}, h_{0}\right)+ & c_{0}\left(\sum_{j=1}^{l} \max \left\{f_{j}^{\prime}\left(\bar{x}, h_{0}\right),-f_{j}^{\prime}\left(\bar{x}, h_{0}\right)\right\}\right. \\
& \left.+\sum_{i \in I(\bar{x})} \max \left\{g_{j}^{\prime}\left(\bar{x}, h_{0}\right), 0\right\}\right)<0 .
\end{aligned}
$$

By the definition of quasidifferential there exist $w_{0}^{*} \in \bar{\partial} u(\bar{x}), v_{j}^{*} \in \underline{\partial} f_{j}(\bar{x}), w_{j}^{*} \in$ $\bar{\partial} f_{j}(\bar{x}), j \in J$, and $z_{i}^{*} \in \bar{\partial} g_{i}(\bar{x}), i \in I(\bar{x})$, such that

$$
\begin{aligned}
& u^{\prime}\left(\bar{x}, h_{0}\right)=\max _{v^{*} \in \underline{\partial} u(\bar{x})}\left\langle v^{*}, h_{0}\right\rangle+\left\langle w_{0}^{*}, h_{0}\right\rangle, \\
& f_{j}^{\prime}\left(\bar{x}, h_{0}\right)=\max _{v^{*} \in \underline{\partial} f_{j}(\bar{x})}\left\langle v^{*}, h_{0}\right\rangle+\left\langle w_{j}^{*}, h_{0}\right\rangle, \quad \forall j \in J, \\
& f_{j}^{\prime}\left(\bar{x}, h_{0}\right)=\left\langle v_{j}^{*}, h_{0}\right\rangle+\min _{w^{*} \in \underline{\partial} f_{j}(\bar{x})}\left\langle w^{*}, h_{0}\right\rangle, \quad \forall j \in J, \\
& g_{i}^{\prime}\left(\bar{x}, h_{0}\right)=\max _{v^{*} \in \underline{\partial} g_{i}(\bar{x})}\left\langle v^{*}, h_{0}\right\rangle+\left\langle z_{i}^{*}, h_{0}\right\rangle, \quad \forall i \in I(\bar{x}) .
\end{aligned}
$$

Hence taking into account (21) one obtains that $\xi_{c_{0}}\left(h_{0}\right)<0$, where the function $\xi_{c}$ is defined in the proof of Theorem 3 On the other hand, from the validity of (20) with $\max \left\{\underline{\mu}_{j}+\bar{\mu}_{j}, \lambda_{i}\right\} \leq c_{0}$ it follows that $\mathbb{O} \in \partial \xi_{c_{0}}(\mathbb{O})$ (see the proof of Theorem 3). Therefore $\xi_{c_{0}}(h) \geq \xi_{c_{0}}(\mathbb{O})=0$ for all $h \in X$, which contradicts the inequality $\xi_{c_{0}}\left(h_{0}\right)<0$. Thus, (19) holds true for $c=c_{0}$.

Let us finally show the independence of (20) on the choice of quasidifferentials. Indeed, if (20) is valid for one choice of quasidifferentials of the functions $u, f_{j}$, and $g_{i}$, then, as we have just proved, optimality condition (19) is satisfied. Hence with the use of the implication (19) $\Longrightarrow(20)$ one obtains that (20) is valid for any other choice of quasidifferentials of the functions $u, f_{j}$, and $g_{i}$.

Let us also give a simple example demonstrating that in some cases the optimality conditions from Theorem 3 are much sharper than optimality conditions in terms of various subdifferentials.

Example 5. Let $X=\mathbb{R}^{2}$, and consider the following optimization problem:

$$
\min u(x)=-x_{1}+x_{2} \quad \text { subject to } f_{1}(x)=\left|x_{1}\right|-\left|x_{2}\right|=0 .
$$

Put $\bar{x}=\mathbf{0}_{2}$. Observe that $\bar{x}$ is not a locally optimal solution of problem (22), since for any $t>0$ the point $x(t)=(t,-t)$ is feasible for this problem and $u(x(t))=-2 t<0=u(\bar{x})$. Nevertheless, let us verify that several subdifferentialbased optimality conditions fail to disqualify $\bar{x}$ as a non-optimal solution.

We start with necessary optimality conditions in terms of the subdifferential of Michel-Penot [20, which we denote by $\partial_{M P}$. Let $L(x, \lambda)=u(x)+\lambda f_{1}(x)$ 
be the Lagrangian function for problem (22). For any $h \in \mathbb{R}^{2}$ the Michel-Penot directional derivative of $L(\cdot, \lambda)$ at $\bar{x}$ has the form

$$
\begin{aligned}
& d_{M P} L(\cdot, \lambda)[\bar{x}, h]=\sup _{e \in \mathbb{R}^{2}} \limsup _{t \rightarrow+0} \frac{L(x+t(h+e))-L(x+t e)}{t} \\
= & \sup _{e \in \mathbb{R}^{2}}\left\{-h_{1}+h_{2}+\lambda\left(\left|h_{1}+e_{1}\right|-\left|e_{1}\right|-\left|h_{2}+e_{2}\right|+\left|e_{2}\right|\right)\right\}=-h_{1}+h_{2}+|\lambda|\left(\left|h_{1}\right|+\left|h_{2}\right|\right) .
\end{aligned}
$$

Hence the Michel-Penot subdifferential of $L(\cdot, \lambda)$ at $\bar{x}$ has the form

$\partial_{M P} L(\cdot, \lambda)(\bar{x})=\partial \varphi\left(\mathbf{0}_{2}\right)=\operatorname{co}\left\{\left(\begin{array}{c}|\lambda|-1 \\ |\lambda|+1\end{array}\right)\left(\begin{array}{c}|\lambda|-1 \\ -|\lambda|+1\end{array}\right)\left(\begin{array}{c}-|\lambda|-1 \\ |\lambda|+1\end{array}\right)\left(\begin{array}{c}-|\lambda|-1 \\ -|\lambda|+1\end{array}\right)\right\}$

where $\varphi(h)=d_{M P} L(\cdot, \lambda)[\bar{x}, h]$. Consequently, for any $\lambda \in \mathbb{R}$ such that $|\lambda| \geq 1$ one has $\mathbf{0}_{2} \in \partial_{M P} L(\cdot, \lambda)(\bar{x})$, which implies that the optimality conditions from [20] are satisfied at $\bar{x}$. Furthermore, note that $\partial_{M P} L(\cdot, \lambda)(\bar{x})=\partial_{C l} L(\cdot, \lambda)(\bar{x})$, which implies that optimality conditions in terms of the Clarke subdifferential [5, Theorem 6.1.1] are satisfied at $\bar{x}$ for any $\lambda$ with $|\lambda| \geq 1$ as well.

Next, we consider optimality conditions in term of the Jeyakumar-Luc subdifferential [39], which we denote by $\partial_{J L}$. By [39, Example 2.1] one has $\partial_{J L} f_{1}(\bar{x})=\left\{(1,-1)^{T},(-1,1)^{T}\right\}$, and clearly $\partial_{J L} u(\bar{x})=\left\{(-1,1)^{T}\right\}$. Hence for any $\lambda \in \mathbb{R}$ with $|\lambda| \geq 1$ one has $\mathbf{0}_{2} \in \partial_{J L} u(\bar{x})+\lambda \operatorname{co} \partial_{J L} f_{1}(\bar{x})$, i.e. the optimality conditions in terms of the Jeyakumar-Luc subdifferential [39, Corollary 3.4] are satisfied at $\bar{x}$.

Let us now consider optimality conditions in terms of approximate (graded, Ioffe) subdifferentials (see [21, 23, 32]), which we denote by $\partial_{a}$. Observe that for any $x \in \mathbb{R}^{2}$ such that $x_{1}, x_{2}>0$ one has $L(x, 1)=0$, which obviously implies that $\partial_{x}^{-} L(x, 1)=\left\{\mathbf{0}_{2}\right\}$ for any such $x$, where $\partial_{x}^{-} L(x, 1)$ is the Dini subdifferential of $L(\cdot, 1)$ at $x$. Therefore, $\mathbf{0}_{2} \in \partial_{a} L(\cdot, 1)(\bar{x})=\limsup _{x \rightarrow \bar{x}} \partial_{x}^{-} L(x, 1)$, i.e. the optimality conditions in terms of approximate subdifferential [21, Proposition 12] are satisfied at $\bar{x}$ (here lim sup is the outer limit).

Let us also consider optimality conditions in terms of the Mordukhovich basic subdifferential [31, which we denote by $\partial_{M}$. One can check (see [30, p. 92-93]) that

$$
\partial_{M} f_{1}(\bar{x})=\operatorname{co}\left\{\left(\begin{array}{c}
1 \\
-1
\end{array}\right),\left(\begin{array}{l}
-1 \\
-1
\end{array}\right)\right\} \cup \operatorname{co}\left\{\left(\begin{array}{l}
1 \\
1
\end{array}\right),\left(\begin{array}{c}
-1 \\
1
\end{array}\right)\right\} .
$$

Therefore, $-\nabla u(\bar{x}) \in \partial_{M} f_{1}(\bar{x})$, i.e. the optimality conditions in terms of the Mordukhovich basic subdifferential [31, Theorem 5.19] hold true at $\bar{x}$.

Finally, let us verify that optimality conditions (20) from Theorem 3 are not satisfied at $\bar{x}$, i.e. unlike optimality conditions in terms of various subdifferentials, optimality conditions based on quasidifferentials detect the non-optimality of $\bar{x}$.

Arguing by reductio ad absurdum, suppose that (20) holds true. Then for $v_{1}^{*}=(1,0)^{T} \in \underline{\partial} f_{1}(\bar{x})$ and $w_{1}^{*}=(0,1)^{T} \in \bar{\partial} f_{1}(\bar{x})$ (see Example 3) there exist $\underline{\mu}_{1}, \bar{\mu}_{1} \geq 0$ such that

$$
0 \in\left(\begin{array}{c}
-1 \\
1
\end{array}\right)-\underline{\mu}_{1} \operatorname{co}\left\{\left(\begin{array}{c}
1 \\
-1
\end{array}\right),\left(\begin{array}{l}
1 \\
1
\end{array}\right)\right\}+\bar{\mu}_{1} \operatorname{co}\left\{\left(\begin{array}{c}
-1 \\
1
\end{array}\right),\left(\begin{array}{l}
1 \\
1
\end{array}\right)\right\}
$$

or equivalently

$$
-1-\underline{\mu}_{1}-\bar{\mu}_{1} \leq 0 \leq-1-\underline{\mu}_{1}+\bar{\mu}_{1}, \quad 1+\bar{\mu}_{1}-\underline{\mu}_{1} \leq 0 \leq 1+\bar{\mu}_{1}+\underline{\mu}_{1} .
$$


From the third inequality it follows that $1+\overline{\mu_{1}} \leq \mu_{1}$, while from the second inequality it follows that $1+\underline{\mu}_{1} \leq \bar{\mu}_{1}$. Therefore $2+\bar{\mu}_{1} \leq \underline{-}_{1}$, which is impossible. Thus, optimality conditions (20) do not hold true at $\bar{x}$.

As was shown in Remark 4, the function $f_{1}$ is metrically regular near the point $(\bar{x}, 0)$, which obviously implies that the penalty term $\varphi(x)=\left|f_{1}(x)\right|$ has a local error bound at $\bar{x}$. Therefore, by Theorem 3 and Remark 8 one can conclude that optimality conditions (20) are not satisfied at $\bar{x}$ due to the non-optimality of this point.

\section{References}

[1] A. Auslender. Stability in mathematical programming with nondifferentiable data. SIAM J. Control Optim., 22:239-254, 1984.

[2] D. Azé. A unified theory for metric regularity of multifunctions. J. Convex Anal., 13:225-252, 2006.

[3] J. F. Bonnans and A. Shapiro. Perturbation analysis of optimization problems. Springer Science+Business Media, New York, 2000.

[4] J. M. Borwein. Stability and regular point of inequality systems. J. Optim. Theory Appl., 48:9-52, 1986.

[5] F. H. Clarke. Optimization and Nonsmooth Analysis. Wiley-Interscience, New York, 1983.

[6] R. Cominetti. Metric regularity, tangent sets, and second-order optimality conditions. Appl. Math. Optim., 21:265-287, 1990.

[7] V. F. Demyanov. Fixed point theorem in nonsmooth analysis and its applications. Numer. Funct. Anal. Optim., 16:53-109, 1995.

[8] V. F. Demyanov. Exhausters and convexificators - new tools in nonsmooth analysis. In V. Demyanov and A. Rubinov, editors, Quasidifferentiability and Related Topics, pages 85-137. Kluwer Academic Publishers, Dordrecht, 2000 .

[9] V. F. Demyanov and V. Jeyakumar. Hunting for a smaller convex subdifferential. J. Glob. Optim., 10:305-326, 1997.

[10] V. F. Demyanov and A. M. Rubinov. Constructive Nonsmooth Analysis. Peter Lang, Frankfurt am Main, 1995.

[11] V. F. Demyanov and A. M. Rubinov, editors. Quasidifferentiability and Related Topics. Kluwer Academic Publishers, Dordrecht, 2000.

[12] M. V. Dolgopolik. Abstract convex approximations of nonsmooth functions. Optim., 64:1439-1469, 2015.

[13] M. V. Dolgopolik. A unifying theory of exactness of linear penalty functions. Optim., 65:1167-1202, 2016.

[14] M. V. Dolgopolik. A convergence analysis of the method of codifferential descent. Comput. Optim. Appl., 71:879-913, 2018. 
[15] S. I. Dudov. Subdifferentiability and superdifferentiability of distance functions. Math. Notes, 4:440-450, 1997.

[16] I. Ekeland and R. Temam. Convex Analysis and Variational Problems. SIAM, Philadelphia, 1999.

[17] Y. Gao. Demyanov difference of two sets and optimality conditions of Lagrange multiplier type for constrained quasidifferentiable optimization. J. Optim. Theory Appl., 104:377-394, 2000.

[18] F. Giannessi. A common understanding or a common misunderstanding? Numer. Funct. Anal. Optim., 16:1359-1363, 1995.

[19] B. M. Glover. On quasidifferentiable functions and non-differentiable programming. Optim., 24:253-268, 1992.

[20] A. Ioffe. A Lagrange multiplier rule with small convex-valued subdifferentials for nonsmooth problems of mathematical programming involving equality and nonfunctional constraints. Math. Program., 58:137-145, 1993.

[21] A. D. Ioffe. Approximate subdifferentials and applications I: the finite dimensional theory. Trans. Am. Math. Soc., 281:389-416, 1984.

[22] A. D. Ioffe. Metric regularity and subdifferential calculus. Russ. Math. Surv., 55:501-558, 2000.

[23] A. D. Ioffe. On the theory of subdifferentials. Adv. Nonlinear Anal., 1:47$120,2012$.

[24] A. D. Ioffe. Variational Analysis of Regular Mappings: Theory and Applications. Springer Intenational Publishing, Cham, 2017.

[25] V. Jeyakumar and D. T. Luc. Nonsmooth calculus, minimality, and monotonicity of convexificators. J. Optim. Theory Appl., 101:599-621, 1999.

[26] L. Kuntz. A charaterization of continuously codifferentiable function and some consequences. Optim., 22:539-547, 1991.

[27] L. Kuntz and S. Scholtes. Constraint qualifications in quasidifferentiable optimization. Math. Program., 60:339-347, 1993.

[28] B. Luderer. Does the special choice of quasidifferentials influence necessary minimum conditions? In W. Oettli and D. Pallaschke, editors, Advances in Optimization, pages 256-266. Springer-Verlag, Berlin, Heidelberg, 1992.

[29] B. Luderer, R. Rösiger, and U. Würker. On necessary minimum conditions in quasidifferential calculus: independence of the specific choice of quasidifferentials. Optim., 22:643-660, 1991.

[30] B. S. Mordukhovich. Variational Analysis and Generalized Differentiation I: Basic Theory. Springer-Verlag, Berlin, Heidelberg, 2006.

[31] B. S. Mordukhovich. Variational Analysis and Generalized Differentiation II: Applications. Springer-Verlag, Berlin, Heidelberg, 2006. 
[32] J.-P. Penot. Calculus Without Derivatives. Springer Science+Business Media, New York, 2013.

[33] L. N. Polyakova. On the minimization of a quasidifferentiable function subject to equality-type quasidifferentiable constraints. In V. F. Demyanov and L. C. W. Dixon, editors, Quasidifferential Calculus, pages 44-55. Springer, Berlin, Heidelberg, 1986.

[34] A. Shapiro. On optimality conditions in quasidifferentiable optimization. SIAM J. Control Optim., 22:610-617, 1984.

[35] A. Shapiro. Quasidifferential calculus and first-order optimality conditions in nonsmooth optimization. In V. F. Demyanov and L. C. W. Dixon, editors, Quasidifferential Calculus, pages 56-68. Springer, Berlin, Heidelberg, 1986.

[36] A. Uderzo. Fréchet quasidifferential calculus with applications to metric regularity of continuous maps. Optim., 54:469-493, 2005.

[37] A. Uderzo. Stability properties of quasidifferentiable systems. Vestn. St. Petesb. Univ. Ser. 10. Appl. Math., Inform., Control Process., 3:70-84, 2006. [in Russian]. Available online at: $\quad$ https://cyberleninka.ru/article/v/svoystva-ustoychivosti-dlyakvazidifferentsiruemyh-sistem-1.

[38] A. Uderzo. Convex difference criteria for the quantitative stability of parametric quasidifferentiable systems. Set-Valued Anal., 15:81-104, 2007.

[39] X. Wang and V. Jeyakumar. A sharp Lagrange multiplier rule for nonsmooth mathematical programming problems involving equality constraints. SIAM J. Optim., 10:1136-1148, 2000. 